\title{
ISL1 is an essential determinant of structural and functional tonotopic representation of sound
}

3

4

Authors: Iva Filova ${ }^{\mathrm{a},}{ }^{1}$, Kateryna Pysanenko ${ }^{\mathrm{b}, 1}$, Mitra Tavakolia Simona Vochyanova ${ }^{\mathrm{a}}$, Martina Dvorakova ${ }^{a}$, Romana Bohuslavova ${ }^{a}$, Ondrej Smolik ${ }^{a}$, Sarka Benesova ${ }^{c}$, Lukas Valihrach $^{\mathrm{c}}$, Ebenezer N. Yamoah ${ }^{\mathrm{d}}$, Josef Syka ${ }^{\mathrm{b}, 2}$, Bernd Fritzsch ${ }^{\mathrm{e}, 2}$, and Gabriela Pavlinkova ${ }^{\mathrm{a}, 2,{ }^{*}}$

\section{Affiliations:}

aLaboratory of Molecular Pathogenetics, Institute of Biotechnology CAS, 25250 Vestec,

\section{Czechia}

${ }^{b}$ Department of Auditory Neuroscience, Institute of Experimental Medicine CAS, 14220

Prague, Czechia

'Laboratory of Gene Expression, Institute of Biotechnology CAS, 25250 Vestec, Czechia

${ }^{\mathrm{d} D}$ Department of Physiology, School of Medicine, University of Nevada Reno, NV 89557, USA

eDepartment of Biology, Department of Otolaryngology, University of Iowa, Iowa City, IA 52242-1324, USA

${ }^{1}$ I.F. and K.P. contributed equally to this work.

${ }^{2}$ contributed to the conceptualization, evaluation, and writing of this report.

* to whom correspondence should be addressed.

Email: Gabriela.Pavlinkova@ibt.cas.cz 


\section{Abstract}

24 A cardinal feature of the auditory pathway is frequency selectivity, represented in the form of a tonotopic map from the cochlea to the cortex. The molecular determinants of the auditory frequency map are unknown. Here, we discovered that the transcription factor ISL1 regulates molecular and cellular features of auditory neurons, including the formation of the spiral ganglion, and peripheral and central processes that shape the tonotopic representation of the auditory map. We selectively knocked out Isll in auditory neurons using Neurod $1^{\mathrm{Cre}}$ strategies. In the absence of Isll, spiral ganglion neurons migrate into the central cochlea and beyond, and the cochlear wiring is profoundly reduced and disrupted. The central axons of Isll mutants lose their topographic projections and segregation at the cochlear nucleus. Transcriptome analysis of spiral ganglion neurons shows that $I s l l$ regulates neurogenesis, axonogenesis, migration, neurotransmission-related machinery, and synaptic communication patterns. We show that peripheral disorganization in the cochlea affects the physiological properties of hearing in the midbrain and auditory behavior. Surprisingly, auditory processing features are preserved despite the significant hearing impairment, revealing central auditory pathway resilience and plasticity in Isll mutant mice. Mutant mice have a reduced acoustic startle reflex, altered prepulse inhibition, and characteristics of compensatory neural hyperactivity centrally. Our findings show that ISL1 is one of the obligatory factors required to sculpt auditory structural and functional tonotopic maps. Still, upon Isll deletion, the ensuing central compensatory

\section{Keywords}

48 plasticity of the auditory pathway does not suffice to overcome developmentally induced peripheral dysfunction of the cochlea.

spiral ganglion neurons, inferior colliculus, auditory nuclei, inner ear, brain plasticity, Isll mutation, auditory maps, auditory behavior 


\section{Introduction}

Spiral ganglion neurons (SGNs) are bipolar, extending peripheral processes to the hair cells within the sensory epithelium (the organ of Corti) and central axons towards the cochlear nucleus $(\mathrm{CN})$ complex, the first auditory nuclei in the brain. Sound-induced vibrations that reach the cochlea are amplified by the outer hair cells (OHCs) organized in three rows and innervated by type II SGNs. The inner hair cells (IHCs) receive, transduce, and transmit the auditory signal to type I SGNs that convey the signal via the vestibulocochlear cranial nerve to the $\mathrm{CN}$ of the brainstem. The auditory neurons are organized within the cochlea in an orderly fashion according to the frequency with high frequencies at the base and low frequencies at the apex ${ }^{1,2}$. The cochleotopic or tonotopic pattern is maintained throughout the auditory pathways in the brain ${ }^{3}$. The central auditory pathway transmits ascending acoustic information from the $\mathrm{CN}$ through the lateral lemniscus complex, the inferior colliculus in the midbrain, the medial geniculate nucleus of the thalamus to the auditory cortex ${ }^{4}$. The efferent motor neurons consist of the medial olivocochlear motor neurons, which modulate the cochlear sound amplification by $\mathrm{OHCs}$, whereas lateral olivocochlear motor neurons innervate afferent sensory neurons and regulate cochlear nerve excitability ${ }^{4,5,6}$.

The cellular and molecular regulation of neuronal migration and the establishment of tonotopic connections to the hair cells or neurons of the hindbrain's first auditory nuclei, the $\mathrm{CN}$, are not fully understood. Several transcription factors govern the development of inner ear neurons, including NEUROG1 ${ }^{7}$, NEUROD ${ }^{8}{ }^{8}$, GATA3 ${ }^{9,10}$, and POU4F $1{ }^{11}$. However, how the transcriptional networks regulate SGN development and how abnormalities in auditory neurons and neuronal wiring reshape the auditory central pathways' assembly and acoustic information processing remain unresolved.

The transcription factor Neurod 1 plays a vital role in the differentiation and survival of inner ear neurons ${ }^{12,13}$. We previously demonstrated that $I s l I^{\mathrm{Cre}}$-mediated Neurodl deletion (Neurod1CKO) resulted in a disorganized cochleotopic projection from SGNs ${ }^{14}$, affecting acoustic information processing in the central auditory system of adult mice at the physiological and behavioral levels ${ }^{15}$. During ear development, ISL1 is expressed in the differentiating neurons and sensory precursors $14,16,17,18,19$. Isll is expressed in all four populations of SGNs (type Ia, Ib, Ic, and type II) identified by single-cell RNA sequencing ${ }^{20}$. Studies suggest that ISL1 plays a role in developing neurons and sensory cells, but a direct evaluation of ISL1 inner ear functions remains unknown. Using Neurod $1^{\text {Cre } 21}$, we generated a mutant with a conditional deletion of Isll (IsllCKO), eliminating Isll in neurons without directly affecting the development of the inner ear sensory epithelium. We first provide genetic and functional evidence for ISL1's role in establishing spiral ganglion peripheral projection map and proper central auditory circuitry. Most Isl ICKO neurons migrated into the center of the cochlear modiolus. They extended outside the cartilaginous otic capsule, in contrast, to control animals with the arrangement of SGNs in parallel to the spiraling cochlear duct. Additionally, we analyzed a transcriptome of neurons, hearing function, sound information processing in the inferior colliculus, and auditory behavior of IsllCKO to demonstrate how the abnormal development of SGNs affects the formation, wiring, and operation of the auditory sensory maps.

\section{Results}

The absence of ISL1 causes disorganized innervation and abnormal location of neurons in the cochlea

To investigate the role of ISL1 in inner ear neuron development, we eliminated Isl 1 by crossing Is $l^{\text {loxPloxP }}$ mice ${ }^{22}$ with Neurod $I^{\text {Cre }}$ mice ${ }^{21}$. Neurod $I^{\text {Cre }}$ is expressed in sensory neurons but not in the sensory epithelium, as visualized by tdTomato reporter expression (Additional file 1: 
Fig. S1). Analyses of ISL1 expression in IsllCKO confirmed efficient recombination by Neurod $1^{\text {Cre }}$ with virtually no expression of ISL1 already during the differentiation of neurons in the inner ear ganglion as early as embryonic day E10.5, and later in the cochlear neurons (Additional file 1: Fig. S2). It is worth mentioning that no difference was observed in the density of Schwann cells between the spiral ganglia of Isl1CKO and control mice (Additional file 1: Fig. S2C', D').

The overall organization of sensory epithelium in the organ of Corti of IsllCKO was comparable to controls with three rows of OHCs and one row of IHCs (Fig. 1A-E'), as shown by immunolabeling with a hair cell differentiation marker, Myo7a. Whole-mount anti-tubulin staining of innervation showed reduced and disorganized radial fibers. More significant gaps were found between radial fiber bundles, crisscrossing fibers, and unusual dense innervation in the apex, with some large fiber bundles bypassing the organ of Corti and extending to the lateral wall in the $I s l 1 C K O$ cochlea (arrowhead in Fig. 1E'). Note, efferent fibers did not correctly form an intraganglionic spiral bundle (IGSB) in IsllCKO.

Next, we evaluated the effects of Isll deletion on the formation of the spiral ganglion. In control animals, the ganglion was arranged in a spiral parallel to the cochlear duct in the basal and apical half of the cochlea (Fig. 1F, G). In contrast, several SGNs in Isl1CKO accumulated beyond the Rosenthal's canal in the center of the basal turn, in the modiolus (arrowhead in Fig. 1H) that usually carries only afferent and efferent fibers. Only a few clusters of neurons were found in the apex (Fig. 1I). The unusual accumulation of cochlear neurons intertwined with the central axons in the modiolus of Isl1CKO compared to the control mice is depicted in the immunolabeled sections of the cochlea (Fig. 1J, K). A significantly smaller proportion of neurons located in the regular position parallel to the organ of Corti in the Rosental's canal of IsllCKO compared to the strip of SGNs in the control cochlea (Fig. 1L). In line with the unusual position of SGNs in the IsllCKO cochlea, much elongated and disorganized radial fibers extended from the modiolus to the organ of Corti compared to the control cochlea. Note, efferent bundles of IGSB crossing the radial fibers towards IHCs in IsllCKO (arrows in Fig. 1H). The radial fibers were significantly lengthened in all sections of the Isl1CKO cochlea (the apex, mid-apex, mid-base, and base; Additional file 1: Fig. S3), but overall radial fiber density was reduced (Fig. 1M). Anti-tubulin labeling revealed reduced and disorganized innervation in the region of OHCs in IsllCKO compared to controls (Fig. 1N-Q). In controls, fibers extending through the tunnel of Corti were oriented towards the base to form three parallel outer spiral bundles (arrows in Fig. 1N). In contrast, guiding defects in the extension of these fibers in IsllCKO were obvious with neurites extending with random turns towards the base and the apex (Fig. 1P, Q).

Interestingly, Isll deficiency resulted in the shortening of the cochlea that was on average about $20 \%$ shorter at postnatal day P0 (Fig. 2A-C). Since Neurod ${ }^{\mathrm{Cre}}$ is expressed only in the neurons (Additional file 1: Fig. S1), the shortening of the cochlea appears to be a secondary effect of the abnormalities in neuronal development. A similar phenotype of the truncated growth of the cochlea was reported for Neurodl and Neurogl mutants because of alternations in spatiotemporal gene expression ${ }^{7,14,15,23}$. Correspondingly to truncated cochlear phenotype $^{7,14,15,23}$, detailed analyses showed abnormalities in the epithelium at the apical end with disorganized rows of OHCs and ectopic IHCs among OHCs in the IsllCKO cochlea (Fig. 2D-G).

Altogether, these results demonstrate the prominent effects of Isll deletion on the formation of the spiral ganglion and innervation patterns in the cochlea, and a confounding feature of the truncated cochlea Isl $1 C K O$ indicates an altered spatial base-to-apex organization of structural and physiological characteristics of auditory neurons. 

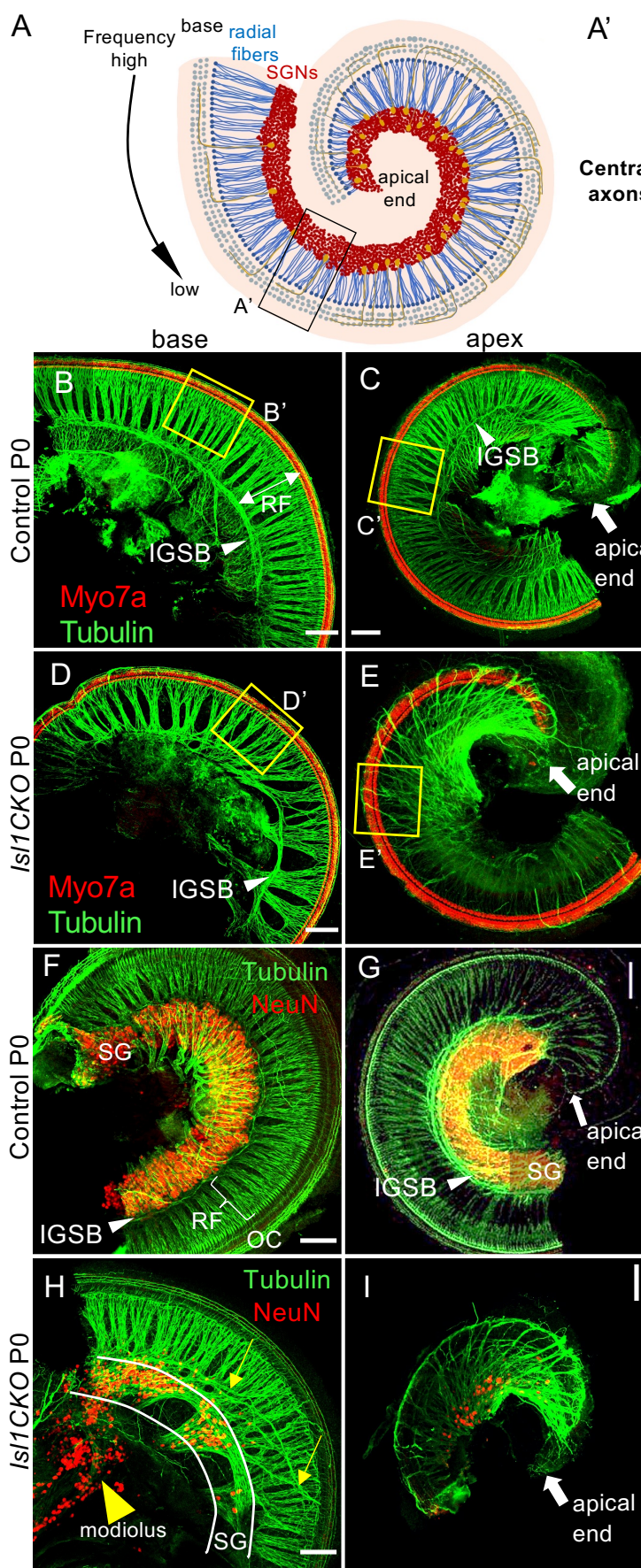

Control base P0

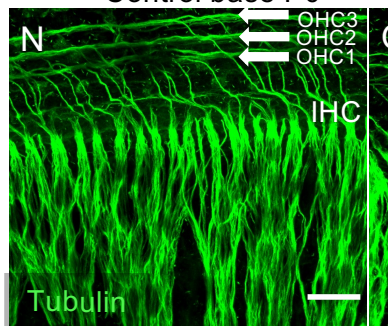

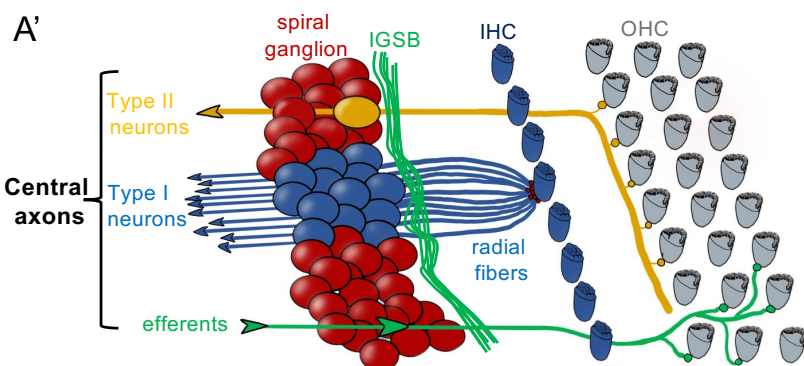
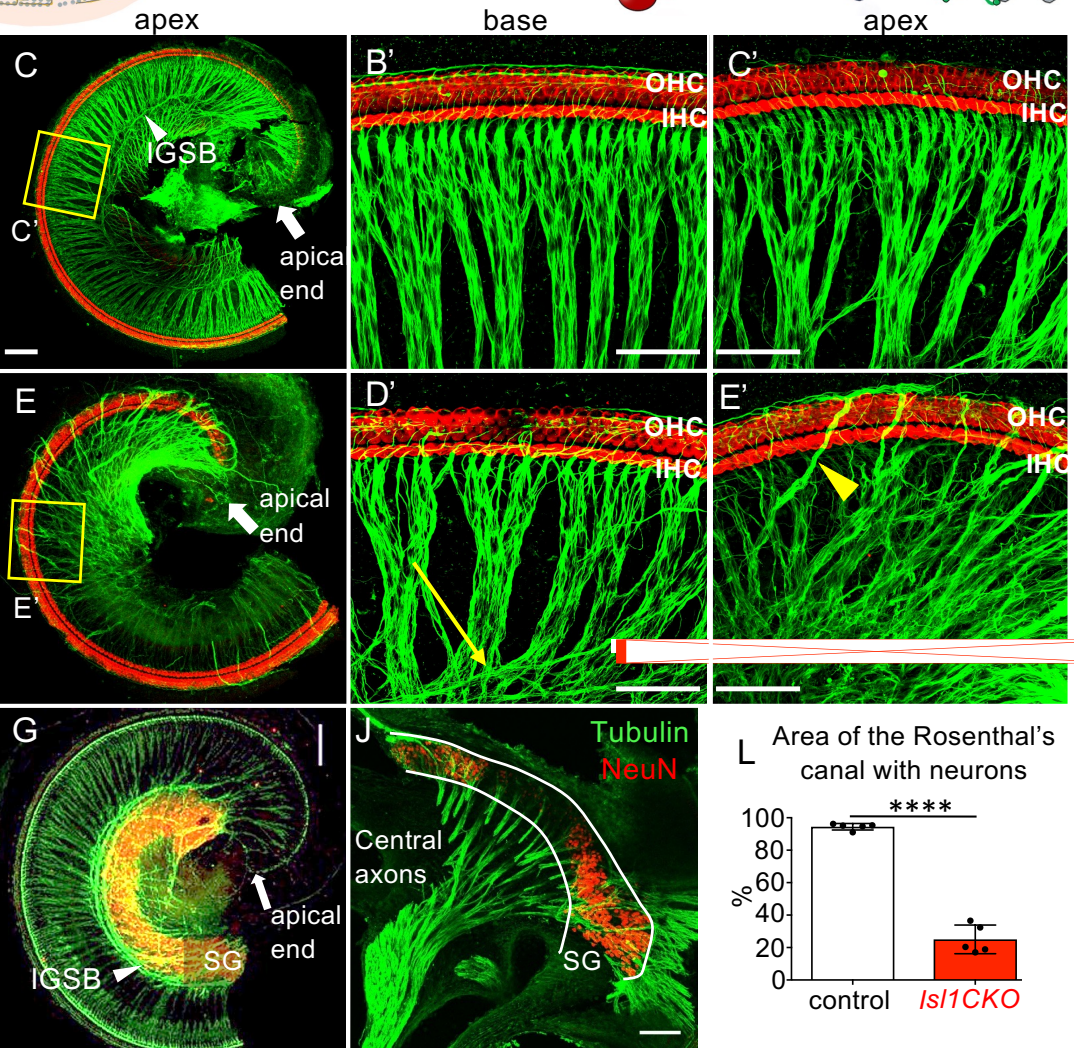

$\mathrm{L}$ Area of the Rosenthal's

canal with neurons

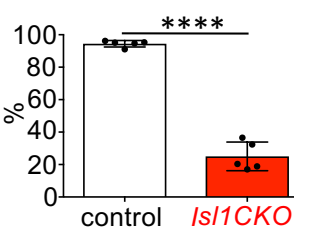

$M_{\text {Density of radial fibers }}$

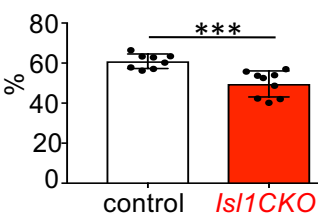

Is/1CKO apex PO

Is/1CKO base P0

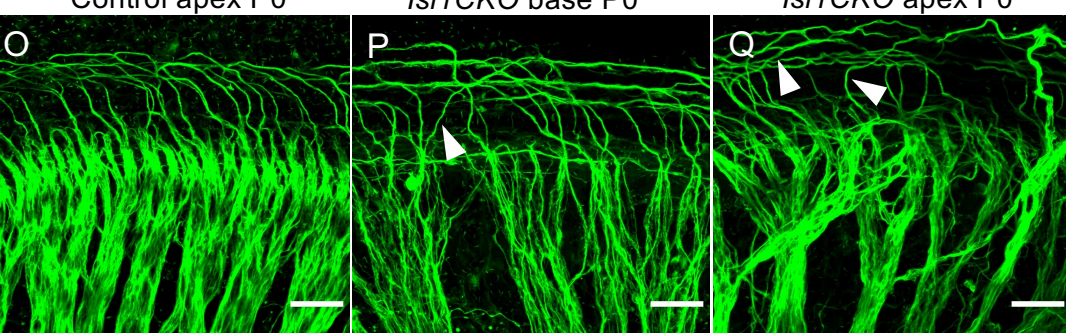

Figure 1. Isl1 deletion results in abnormalities in cochlear innervation and the formation of the spiral ganglion. (A) Diagram of the organization of the cochlea. Spiral ganglion neurons (SGNs) are organized tonotopically from the apex to the base of the cochlea. High-frequency sounds maximally stimulate the base of the cochlea, whereas the largest response to low-frequency sounds occurs in the cochlear apex. (A') The top view diagram onto the sensory epithelium shows outer hair cells (OHCs), inner hair cells (IHCs), and spiral ganglion. Type I neurons extend radial fibers toward IHCs (5-30 neurons innervate one IHC), and type II neurons (representing $5 \%$ of SGNs) receive input from OHCs. The central processes of SGNs relay acoustic information to the brain. (Continued) 


\section{Figure 1 continued}

Efferent axons from the superior olivary complex, forming the intraganglionic spiral bundle (IGSB), innervate OHCs. (BE) Representative images of whole-mount immunolabeling of the cochlear base and apex with anti-Myo7a (a marker of hair cells) and anti-tubulin (nerve fibers) show reduced and disorganized radial fibers (RF), and missing or altered efferent fibers forming IGSB (arrowhead) in Isl1CKO compared to control at postnatal day P0. (B'-E') Higher-magnification images show three rows of OHCs and a row of IHCs forming the organ of Corti and connected to SGNs by RF. Abnormalities in innervation, including larger gaps between radial fiber bundles, crisscrossing fibers (arrows), and fiber bundles bypassing the organ of Corti (arrowheads in E'), are noticeable in the IsllCKO cochlea. (F-I) The shape of the spiral ganglion (SG) is shown in the split basal and apical half of the cochlea in whole-mount immunolabeling with anti-NeuN (a nuclear marker of differentiated neurons) and with anti-tubulin (nerve fibers). In controls, anti-NeuN labeled neurons form a spiral parallel to the organ of Corti (OC) with central axons and RF at P0. In contrast, only a small portion of NeuN ${ }^{+}$neurons are located in the Rosenthal's canal parallel to the OC in $I s l 1 C K O$ (delineated by white lines in $\mathrm{H}$ ). The majority of $\mathrm{NeuN}^{+}$neurons is located in the modiolus (arrowhead). The apical half of the Isl1CKO cochlea contains only clusters of neurons (I). Note disorganized IGSB with fiber bundles directly reaching hair cells (arrows in H) and missing IGSB formation in the apex (I). (J, K) The vibratome sections of the cochlea labeled with anti-tubulin and anti-NeuN show the unusual position of cochlear neurons in the modiolus and neurons entangled in central axons in Isl1CKO compared to control (arrowhead indicates a normal position of the SG). (L) Relative comparison of the area of the spiral ganglion in the Rosenthal's canal containing neurons between control and IsllCKO. The values represent mean $\pm \mathrm{SD}$ (controls $\mathrm{n}=5 ;$ Isl $I C K O \mathrm{n}=5$ ); $t$-test $* * * * \mathrm{P}<0.0001$. (M) The density of radial fibers in the base, mid-base, and mid-apex were lower in the Isl1CKO than in the control cochlea. The values represent mean $\pm \mathrm{SD}(\mathrm{n}=3$ cochlea per genotype); $t$-test $* * * * \mathrm{P}<0.0001$. (N-Q) Highermagnification images show a detail of anti-tubulin labeled innervation with fibers forming three parallel outer spiral bundles (arrows) that innervate multiple OHCs and turning toward the base in the control cochlea. Guiding defects in the extension of these fibers to OHCs are obvious in $I s l 1 C K O$ with some fibers randomly turned toward the apex (arrowheads); note, the disorganization of radial fiber bundles in IsllCKO. Scale bars: $100 \mu \mathrm{m}(\mathrm{B}-\mathrm{K}), 50 \mu \mathrm{m}\left(\mathrm{B}^{\prime}-\mathrm{E}^{\prime}\right), 20 \mu \mathrm{m}(\mathrm{N}-\mathrm{Q})$.

\section{Isl1 regulates neuronal identity and differentiation programs of SGNs}

To gain insight at the molecular level about how the Isll elimination causes the neuronal phenotype in the cochlea, we sought to identify potential ISL1 targets through global transcriptome analysis. We opted to use Bulk-RNA sequencing to obtain sequencing depth and high-quality data ${ }^{24}$. Six biological replicates were used per each genotype, and each replicate contained a total of 100 tdTomato positive SGNs isolated from the cochlea embryonic age E14.5. Spiral ganglia were dissected, dissociated into single cells, and fluorescent tdTomato ${ }^{+}$ cells were FACS-sorted (Fig. 3A). Compared to controls, 650 protein-coding genes were differentially expressed in IsllCKO neurons (adjusted p-value $<0.05$, and fold change $>2$ cutoff values, see Methods), 332 genes down- and 318 genes up-regulated (Fig. 3B, Additional file 2: Table S1). Gene ontology (GO) term enrichment analysis for the GO term category biological process revealed highly enriched GO terms associated with neuron development, including neurogenesis, neuron differentiation, and nervous system development, in both upand down-differentially expressed genes (Fig. 3C, Additional file 2: Table S2, S3). The most enriched and specific GO categories for downregulated genes were associated with neurotransmission-related machinery, such as transmembrane transporter, voltage-gated channel, cation and ion transport, and membrane potential regulation, indicating changes in neuronal cell functions. The analysis identified enrichment of downregulated genes involved

167 in axon development, guidance, axonogenesis, and neuronal migration. These genes included members of all four different classes of axon guidance molecules and their cognate receptors, the ephrins-Eph, semaphorins-plexin, netrin-unc5, and slit-roundabout ${ }^{25}$, for instance, Epha5, Epha4, Pdzrn3, Sema3e, Sema6d, Plxna4, Ntn3, Ntng1, Ntng2, Kirrel3, Unc5b, Unc5c, Slitrk3, and Slitrk1. The neurotrophic tyrosine kinase receptors (Ntrk2 and Ntrk3) and a G-proteincoupled chemokine receptor $(\mathrm{Cxcr} 4)$, important regulators of neuronal migration, were also downregulated ${ }^{26}$. These molecular differences dovetail well with abnormalities in the innervation pattern and SGN migration defects in the Isl 1 CKO cochlea. In contrast, upregulated genes particularly enriched in IsllCKO neurons were associated with "regulation of synapse 
organization, synapse structure or activity" as well as "regulation of synapse assembly", arguing for compensation of dramatic changes in the ability of neurons to form neuronal projections towards their targets. Upregulated genes encoding molecules critical for synapse formation and adhesion included several members of the cadherin superfamily ( $C d h 7, C d h 10$, Pcdh8, Pcdh9, Pcdh10, Pcdh18, and Pcdh19), adhesion-related genes (Ptprd, Ptprs, Cntnap2, Itga4, Itga5), and the ephrin ligands (Efna3, Efna5). Interestingly, "synaptic signaling" was among GO terms with the significant representation in both up- and downregulated genes, suggesting changes in synaptic circuits and neuronal activity. We also found many genes encoding transcription factors and signaling molecules, indicating that ISL1 may act through transcription networks instead of defined target genes. The expression of neural-specific bHLH factors important for differentiation and maturation of neurons was increased in IsllCKO, including members of NeuroD (Neurod6, Neurod2), Nscl (Nhlh2), and Olig (Bhlhe23) families ${ }^{27}$. The increased expression of genes encoding LIM domain proteins, the transcription factors Lhx1, Lhx2, Lmo2, Lmo3, and a core
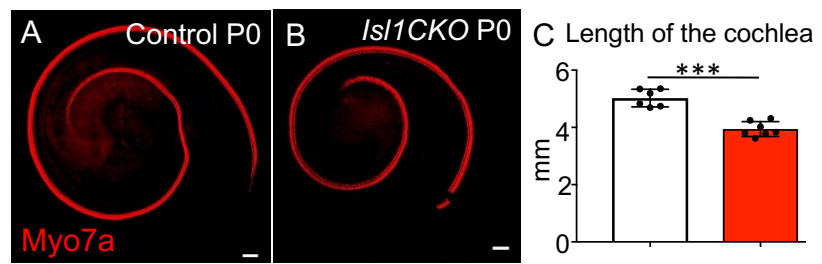

mid-base

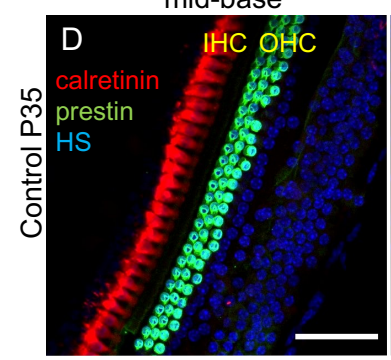

apical end
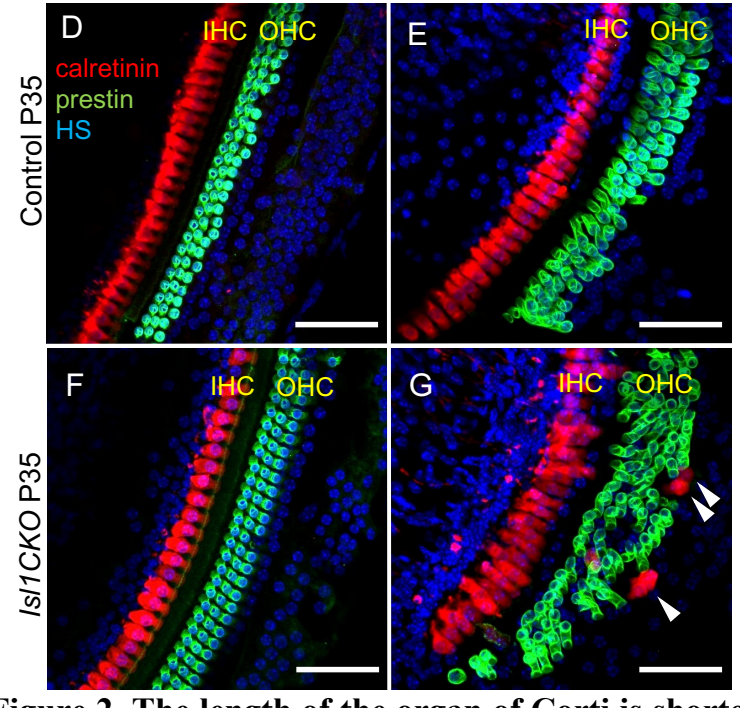

Figure 2. The length of the organ of Corti is shortened in Isl1CKO. (A-C) The organ of Corti of IsllCKO is $20 \%$ shorter than control at $\mathrm{P} 0$, as shown by anti-Myo7a labeling of hair cells. The values represent mean \pm SEM (controls $5.0 \pm 0.1 \mathrm{~mm}, \mathrm{n}=6$; Isl 1 CKO $3.9 \pm 0.1 \mathrm{~mm}, \mathrm{n}=$ 7); $t$-test $* * * \mathrm{P}<0.001$. (D-G) Immunohistochemistry for prestin (a marker for OHCs) and calretinin (a marker for IHCs) shows a comparable cytoarchitecture of the organ of Corti in the cochlear base of controls and IsllCKO but disorganized rows of OHCs in the apical end with multiple $\mathrm{HC}$ rows and ectopic calretinin ${ }^{+} \mathrm{IHCs}$ among OHCs cells (arrowheads) in IsllCKO at P36. HS, Hoechst nuclear staining. Scale bars: $100 \mu \mathrm{m}$ (A, B), $50 \mu \mathrm{m}$ (D-G). member of the PCP signaling paradigm, Prickle1, was detected in IsllCKO neurons. Prickle1 is an important regulator of neuron migration and neurons' distal and central projections in the cochlea ${ }^{28}$. Some of the identified genes encoding regulatory molecules that were shown to be essential for neuronal development, the formation of SGNs, and their projections were downregulated in ISLICKO neurons, such as signaling molecules $S h h^{29}, W n t 3^{30}$, and FgfS $(F g f 10, F g f 11 \text {, and } F g f 13)^{31,32}$, and the transcription factors Gata ${ }^{9}$, 10, Irx 1, Irx 2, Pou $3 f 2$, Pou $42^{18}$, and Runx $1^{20,30}$. Selected differentially expressed genes from the RNAseq analysis, namely $L h x 1, L h x 2, C d h 7, N h l h 2, N t r k 2$, and Ntrk3, were further validated by RT-qPCR of RNA isolated from whole inner ears of E14.5 embryos (Additional file 1: Fig. S4).

Altogether, these changes indicate that ISL1 regulates transcriptional networks that underlie neuronal identity and function during differentiation of SGNs and that Is $I$ elimination results in a major impairment in the development, axonogenesis, migration, and molecular characteristics of these neurons. 
A

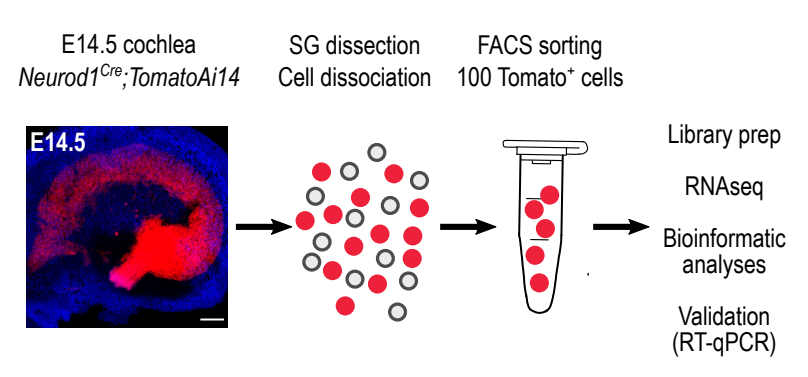

C1

DOWN-regulated gene set enrichment map
B

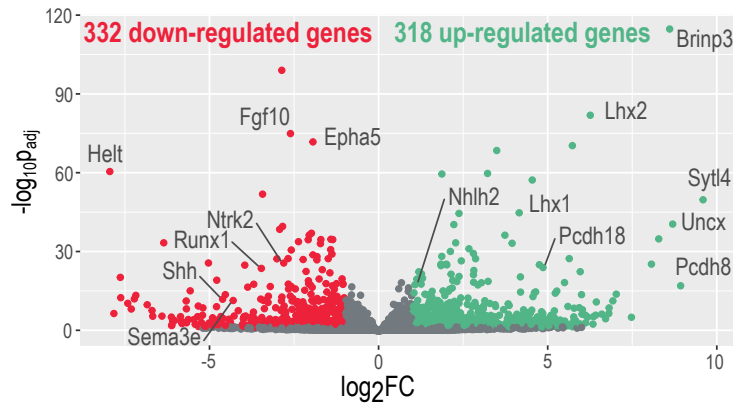

$\mathrm{C} 2$

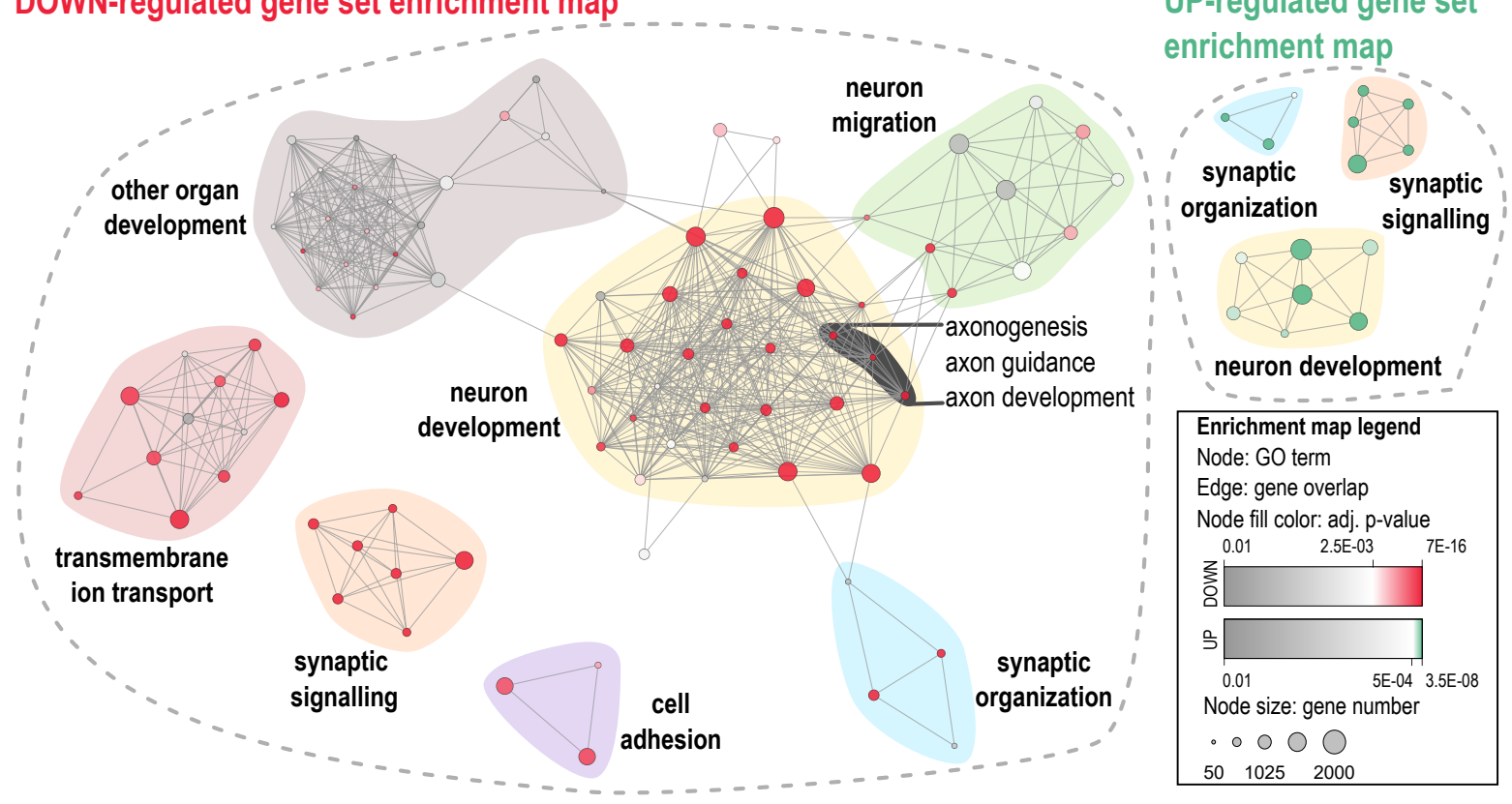

Figure 3. Comparative analysis of control and Isl1CKO transcriptomes. (A) Image of the whole-mount cochlea with genetically labeled tdTomato neurons in the spiral ganglion at E14.5 (HS, Hoechst nuclear staining, Scale bar: $100 \mu \mathrm{m}$ ); and workflow depicts microdissection, dissociation, FACS sorting of single tdTomato ${ }^{+}$spiral ganglion neurons for a bulk of 100 cells RNAseq analysis. (B) The volcano plot shows the change in protein-coding genes' expression level in the Isl1CKO compared to control spiral ganglion neurons (adjusted p-value $<0.05$, and fold change $>2$ cutoff values). The complete list of identified 332 down- and 318 up-differentially expressed genes is in Additional file 2, Table S1. (C) Enrichment map of down- (C1) and up-regulated (C2) gene ontology (GO) sets visualized by the network. Each node represents a GO term; edges depict shared genes between nodes. Node size represents a number of genes of the mouse genome per the GO term, and node fill color represents a GO term significance. Each GO set cluster was assigned with representative keywords; a list of GO sets is available in Additional file 2, Table S2, S3).

\section{Isl1CKO mice have altered hearing function}

224 Considering the substantial abnormalities in the formation of the spiral ganglion, innervation, and molecular neuron features, we assessed the hearing function of IsllCKO mice. We evaluated distortion product otoacoustic emissions (DPOAE) to determine the robustness of $\mathrm{OHC}$ function and cochlear amplification. Otoacoustic emissions are a physiological byproduct of an active amplification mechanism when sound-induced vibrations are amplified in a frequency-specific manner by the OHCs of the organ of Corti ${ }^{33,34}$. Compared to controls, the DPOAE responses of $I$ sl $1 C K O$ were significantly reduced in the frequency range between 4 and $24 \mathrm{kHz}$ (Fig. 4A). DPOAE amplitudes at frequencies of $28 \mathrm{kHz}$ and higher were comparable between control and IsllCKO mice. Based on the physiological place-frequency 


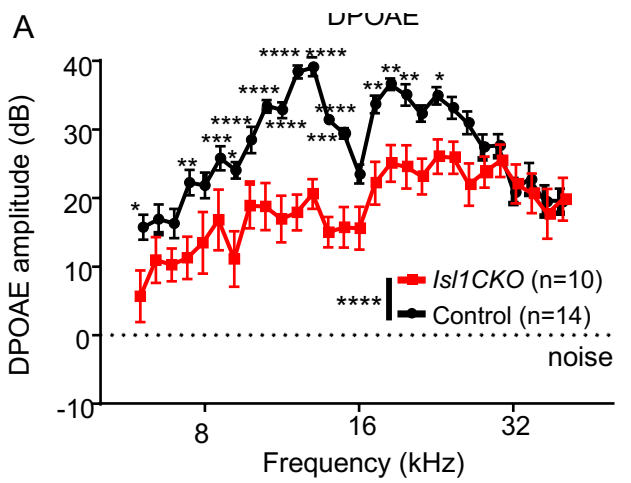

C

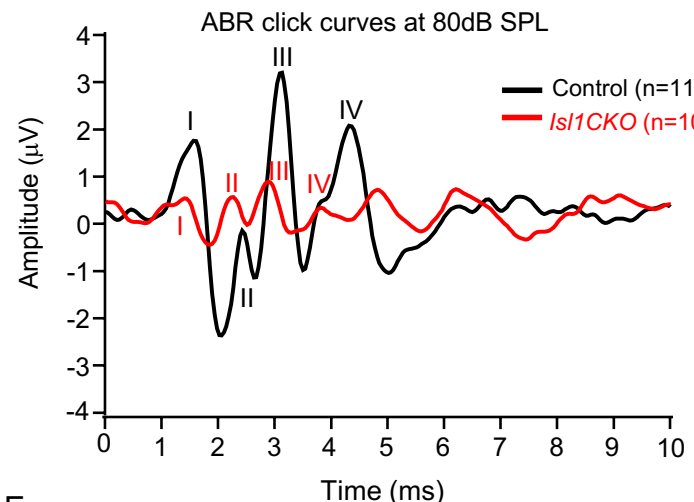

E

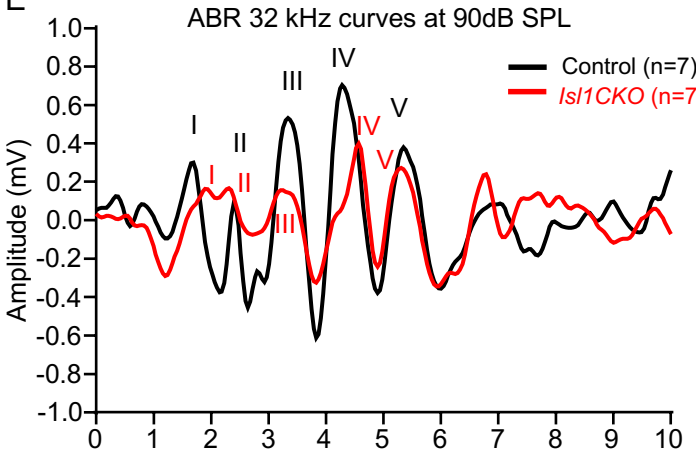

G

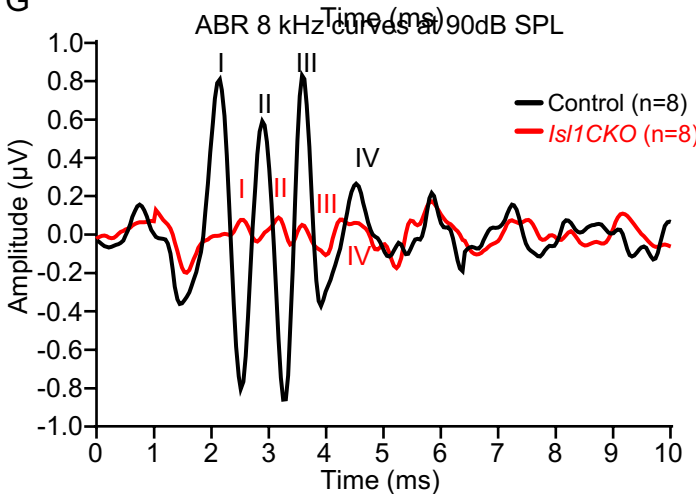

B

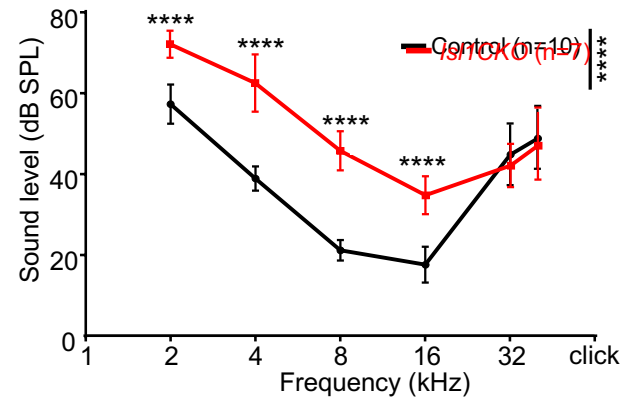

$\mathrm{D}$

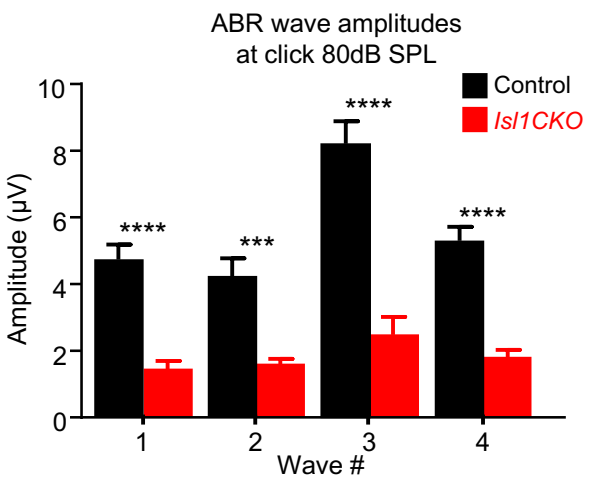

$\mathrm{F}$

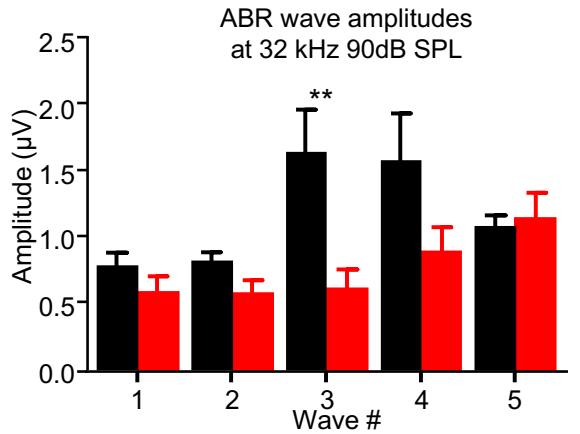

$\mathrm{H}$

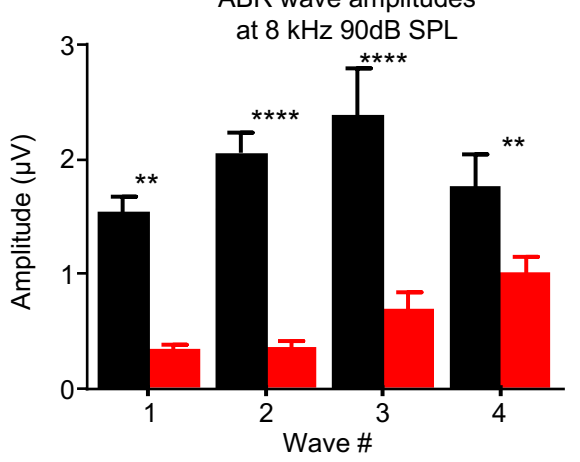

Figure 4. Hearing impairment is detected in IsllCKO mice. (A) Distortion product otoacoustic emissions (DPOAEs) show significantly reduced levels in the low and middle-frequency range (6 - $24 \mathrm{kHz})$. Data are the mean $\pm \mathrm{SEM}$, two-way ANOVA with Bonferroni post-hoc test, $* \mathrm{P}<0.05, * * \mathrm{P}<0.01, * * * \mathrm{P}<0.001 * * * * \mathrm{P}<0.0001$. (B) The average auditory brainstem response (ABR) thresholds of IsllCKO and control mice are analyzed by click-evoked ABR. Data are the mean $\pm \mathrm{SD}$, two-way ANOVA with Bonferroni post-hoc test, $* * * * \mathrm{P}<0.0001$. The graphs show averaged ABR response curves evoked (C) by an $80 \mathrm{~dB}$ SPL click; (E) by a $90 \mathrm{~dB}$ SPL pure tone of $32 \mathrm{kHz}$ frequency; and (G) by a $90 \mathrm{~dB}$ SPL pure tone of $8 \mathrm{kHz}$ frequency. (D, F, H) Averaged individual ABR wave amplitudes are shown for the corresponding peaks. Data are the mean $\pm \mathrm{SEM}$, two-way ANOVA with Bonferroni post-hoc test, $* * \mathrm{P}<0.01 * * * \mathrm{P}<0.001, * * * * \mathrm{P}<0.0001$. 
map in the normal mouse cochlea ${ }^{35}$, frequencies above $28 \mathrm{kHz}$ are located at the basal half of the cochlea from the mid-base to the basal end. These most preserved DPOAE responses correspond to the most close-to-normal distribution of sensory neurons in the cochlear base in IsllCKO mice (Fig. 1H). Thus, decreased DPOAE responses may be attributed to the overall more profound morphological abnormalities in the apex, including a diminished spiral ganglion, disorganized innervation (Fig. 1E, E', I, Q), and disorganized rows of OHCs in the apical end (Fig. 2G).

We evaluated auditory brainstem responses (ABRs), which measure electrical activity associated with the propagation of acoustic information through auditory nerve fibers. Measurements of ABR thresholds showed that all IsllCKO animals displayed elevated thresholds indicative of hearing loss compared to age-matched control animals, except at frequencies above $32 \mathrm{kHz}$, which were comparable to ABR thresholds in control mice (Fig. 4B). Using click-evoked ABR, we evaluated waveform characteristics associated with the propagation of acoustic information through the auditory nerve to higher auditory centers (Fig. 4C). Wave I reflects the synchronous firing of the auditory nerve. In contrast, waves II-V are attributed to the electrical activity of downstream circuits in the $\mathrm{CN}$, superior olivary complex, lateral lemniscus, and inferior colliculus ${ }^{36}$. The amplitudes of ABR waves I-IV were significantly reduced in IsllCKO (Fig. 4D). Since the ABR threshold for $32 \mathrm{kHz}$ and above were comparable between age-matched controls and IsllCKO mutants (Fig. 4B), we used the pure-tone stimuli of $32 \mathrm{kHz}$ to evaluate ABR responses (Fig. 4E). A significant difference amongst the genotypes was only found for amplitude reduction of wave III (Fig. 4F), thus indicating preserved synchronized activities of peripheral and brainstem auditory processing. Although waves I and II amplitude for both mutant and control mice were similar, there were apparent differences in the ABR waveform morphology. The latency of ABR wave I was delayed, the relative interwave latency between peaks I and II was shortened, and the trough between wave I and II diminished, resulting in a fusion of both peaks in Isl1CKO. A delay of the leading peak of ABR wave I recovered towards ABR wave III. The changes in wave I and II characteristics reflect abnormalities in the summated response from SGNs, auditory nerve fibers, and most likely the $\mathrm{CN}$.

Additionally, we used the pure-tone stimulus of $8 \mathrm{kHz}$ to evaluate ABR responses (Fig. $4 \mathrm{G})$, as ABR thresholds for $8 \mathrm{kHz}$ were significantly reduced for Isl1CKO mice. In contrast to the ABR amplitudes at $32 \mathrm{kHz}$ stimuli, the ABR amplitudes for $8 \mathrm{kHz}$ pure-tone stimuli were significantly reduced for all waves (Fig. 4H). The results indicate abnormalities in the cochlear auditory neurons and propagation of acoustic information through auditory nerve fibers to higher auditory centers. We observed a marked drop in the wave I growth function. Still, by comparing wave I and IV peaks, the increased central gain was noted in Isl1CKO (Additional file 1: Fig. S5), indicating compensatory plasticity at higher auditory circuits for cochlear damage with diminished afferent input ${ }^{37}$.

\section{Isl1CKO mice have structural abnormalities in the ascending auditory pathways}

Having recognized the major effects of Isll elimination on the formation of the spiral ganglion and auditory dysfunction, causing sensory hearing loss, as measured by ABRs, we next wanted to establish the morphology of the central auditory pathway components. Using dye tracing, we evaluated the segregation of central axons of the auditory nerve (the cranial nerve VIII; Fig. $5 \mathrm{~A}$, schematic view of dye applications). In controls, the central axons labeled by dyes applied into the cochlear base (red) and apex (green) are segregated in the auditory nerve and from the vestibular ganglion, labeled by dye injected into the vestibular end-organs (magenta, Fig. 5B). In contrast, the central axons from the cochlear base (red) and apex (green) virtually overlapped in the auditory nerve (overlapping yellow fibers), and many neurons labeled by the cochlear dye applications were detected to be intermingled with vestibular neurons in IsllCKO (Fig. 

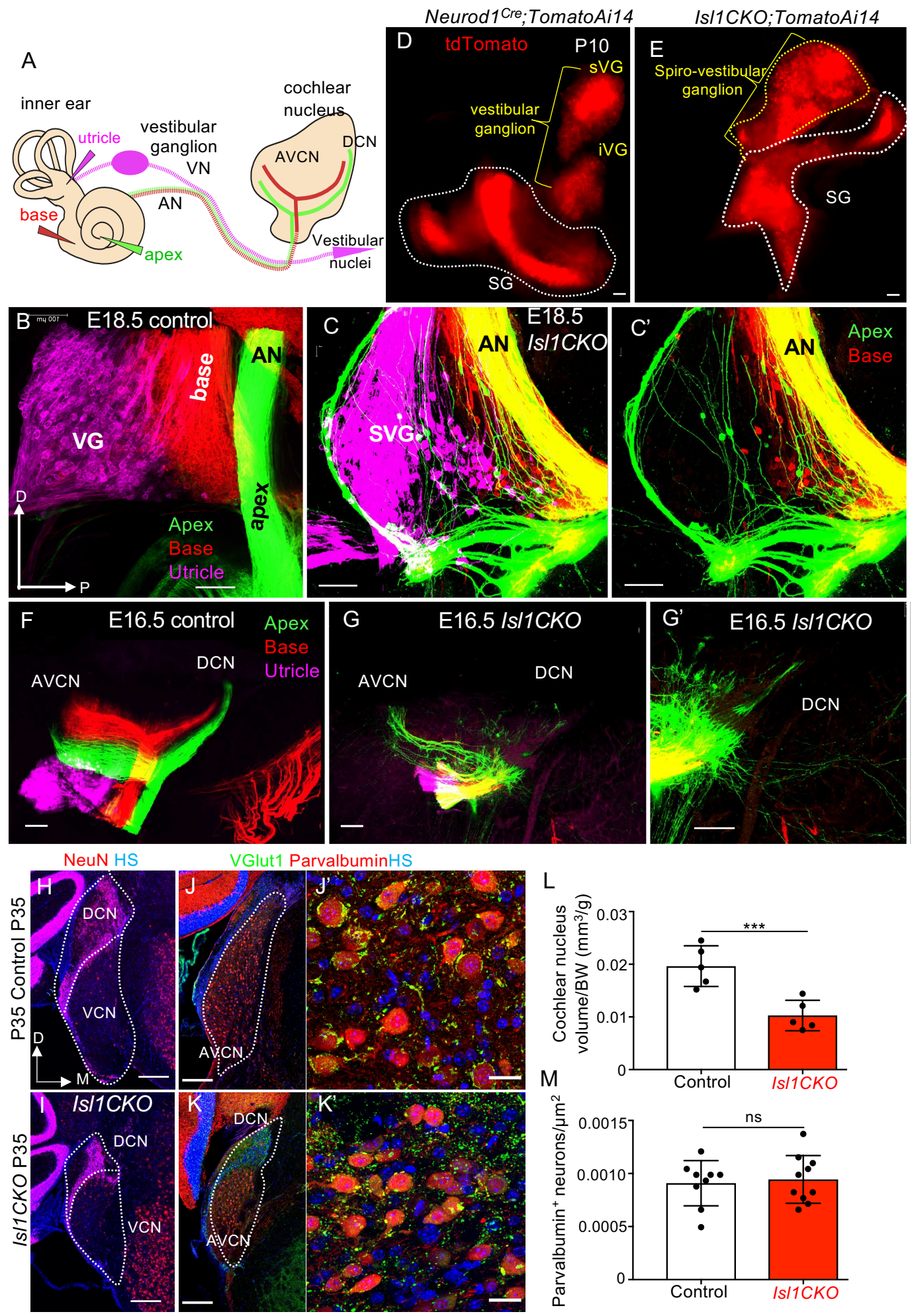

Figure 5. Isl1CKO cochlear neurons project unsegregated and disorganized central projections to the cochlear nucleus. (A) The schematic diagram visualizes dye tracing from the inner ear and its connections to the auditory brainstem, using insertions of differently colored dyes into the vestibular end organ (the utricle, magenta), cochlear base (red), and cochlear apex (green). Axonal projections from cochlear neurons to the cochlear nucleus $(\mathrm{CN})$ bifurcate with one branch synapsing in the dorsal (DCN) and the other innervating the anteroventral $\mathrm{CN}(\mathrm{AVCN})$. (B-C') Injections of different colored dyes (magenta into the utricle, red in the base, green in the apex) label distinct and spatially restricted bundles of neuronal fibers (auditory nerve, $\mathrm{AN}$ ) projecting to the $\mathrm{CN}$ and the vestibular ganglion in controls. In contrast, in IsllCKO, the segregation of central axons is lost, as fibers labeled from the apex (green) and base (red) are completely overlapping in the AN (yellow fibers), and neurons labeled by dyes injected into the cochlear base and apex are mixed with vestibular ganglion neurons (magenta) to form an aberrant enlarged ganglion, the spiro-vestibular ganglion (SVG). A merged image shows apical turn dye and base dye-labeled fibers and soma in the SVG $\left(\mathrm{C}^{\prime}\right)$. (Continued) 


\section{Figure 5 continued}

Note that fibers labeled from the apex (green) form an unusual fiber loop around the SVG in Isl1CKO (C, C'). (D, E) Wholemount of the inner ear with tdTomato reporter labeled neurons shows superior (sVG) and inferior vestibular ganglia (iVG), and the spiral ganglion (SG) in the control reporter mouse. In contrast, vestibular ganglia are fused and enlarged, and the spiral ganglion has lost its spiral shape in $I s l 1 C K O$, shown at postnatal day P10. (F-G') The tonotopic organization of the $\mathrm{CN}$ subdivisions in controls is shown by dye tracing in the AVCN and DCN with low-frequency fibers labeled from the apex (green) and high frequency from the base (red). Projections from the base terminate dorsally to the projections from the apex in the control CN. Central projections of afferents in IsllCKO enter as a single bundle from the apex and base instead of forming separate central projections, with just a few fibers occasionally expanding to the DCN. (H, I) Coronal sections of a brain (immunostained with anti-NeuN, red) of adult littermate controls and Isl1CKO, showing the DCN and $\mathrm{VCN}$; the dotted line indicates the boundaries of the CN. (J, K) Representative images of immunolabeling sections of the brain at P35 using anti-parvalbumin to label the bushy cell soma and anti-VGlut1 to label auditory-nerve endbulbs of Held around the spherical and globular bushy cells. (J', K') Higher-magnification images show the distribution of parvalbumin ${ }^{+}$ neurons and the presence of VGlut $1^{+}$auditory nerve synaptic terminals. (L) Quantification of the adult CN volume, adjusted to body weight and (M) a number of parvalbumin ${ }^{+}$cells per $\mu \mathrm{m}^{2}$ of the AVCN. Data are the mean \pm SD. Two-tailed unpaired t-test $(* * * \mathrm{P}<0.001$; ns, not significant). $\mathrm{D}$, dorsal; $\mathrm{P}$, posterior; $\mathrm{M}$, medial axis. HS, nuclear staining Hoechst. Scale bars: $100 \mu \mathrm{m}\left(\mathrm{B}-\mathrm{G}^{\prime}\right) ; 200 \mu \mathrm{m}(\mathrm{H}-\mathrm{K}) ; 20 \mu \mathrm{m}\left(\mathrm{J}^{\prime}, \mathrm{K}^{\prime}\right)$.

285 5C), forming an aberrant spiro-vestibular ganglion. A merged image shows only apical and

286 basal dye-labeled neuron somas and fibers located in the spiro-vestibular ganglion of Isl1CKO

287 (Fig. 5C'). The unusual fibers labeled by the apical dye application (green) were looping around

288 the vestibular ganglion in IsllCKO (Fig. 5C, $\mathrm{C}^{\prime}$ ). The distinctive shape of inner ear ganglia in

289 IsllCKO was also confirmed by the tdTomato reporter (Fig. 5D, E). Superior and inferior

290 vestibular ganglia, and the spiral ganglion, recognizable as a coil of cochlear auditory neurons,

291 were clearly distinguished in the control reporter mouse. In contrast, the vestibular ganglia

292 were fused and enlarged, and the cochlear ganglion lost its spiral shape in IsllCKO (Fig. 5E).

293 Unfortunately, the mixing of spiral and vestibular ganglion neurons in IsllCKO mice precluded

294 a full quantitative assessment.

295

296

297

The $\mathrm{CN}$ is the first structure of the ascending auditory pathways, where the auditory nerve fibers project. The auditory nerve bifurcated with one branch, synapsing in the posteroventral (PVCN) and dorsal (DCN) $\mathrm{CN}$ and the other innervating the anteroventral $\mathrm{CN}$ (AVCN; Fig. 5A). Dye tracing showed segregated projections of apical and basal cochlear afferents forming parallel isofrequency bands in controls (Fig. 5F). In contrast, comparable injections in IsllCKO showed that axonal projections to the $\mathrm{CN}$ were reduced, restricted, and disorganized and lacked a clear apex and base projection segregations (Fig. 5G). Only a few fibers can occasionally be seen expanding to the DCN in IsllCKO (Fig. 5G'). These results show that tonotopic organization of both the auditory nerve and the $\mathrm{CN}$ is lost in IsllCKO, as the cochlear apex and base projections are not segregated. Since the size and number of neurons in the $\mathrm{CN}$ depend on input from the auditory nerve during a critical development period up to P9 ${ }^{38}$, we also analyzed the volume of the $\mathrm{CN}$ of Isl1CKO. The volume of the $\mathrm{CN}$ of adult mutants was reduced by approximately 50\% compared to controls at postnatal day P35 (Fig. $5 \mathrm{H}, \mathrm{I}, \mathrm{L})$. As $I s l 1$ is not expressed in the $\mathrm{CN}^{15}$, the reduced size is likely a secondary effect associated with reduced afferent input consistent with the impact of neonatal cochlear ablation 310 previously reported ${ }^{2}$.

311 The CN contains a variety of neurons with distinct features. The spherical and globular 312 bushy cells are principal cells that receive large auditory nerve endings, called "endbulbs of 313 Held" and "modified endbulbs," specialized for precise temporal firing 1, 39. Using anti314 parvalbumin to label bushy cell somata and anti-VGlutl to label auditory-nerve endbulbs of 315 Held around the spherical and globular bushy cells ${ }^{40,41}$, we demonstrated that auditory 316 afferents of Isl1CKO and controls formed comparable clusters of boutons that wrap the somas 317 of their targets. Although the $\mathrm{CN}$ of $I s l 1 C K O$ was smaller, the number of parvalbumin ${ }^{+}$neurons 318 per $\mu \mathrm{m}^{2}$ was similar in the VCN of controls and IsllCKO (Fig. 5J-K', M). 


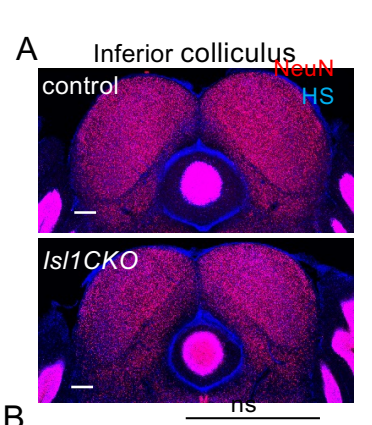

$\mathrm{B}$

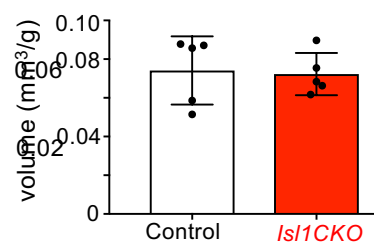

C
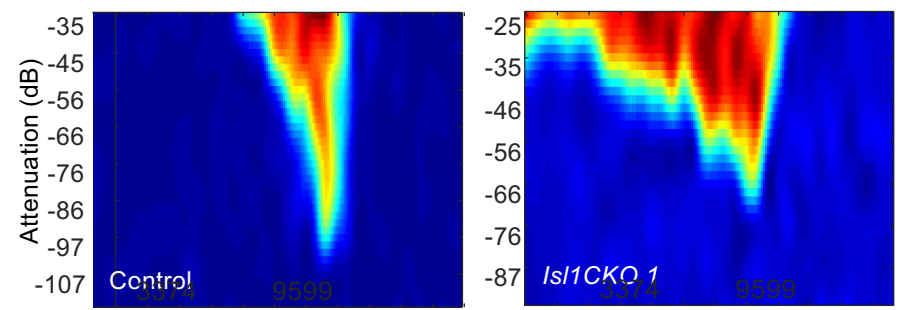

$2000 \quad 5691$
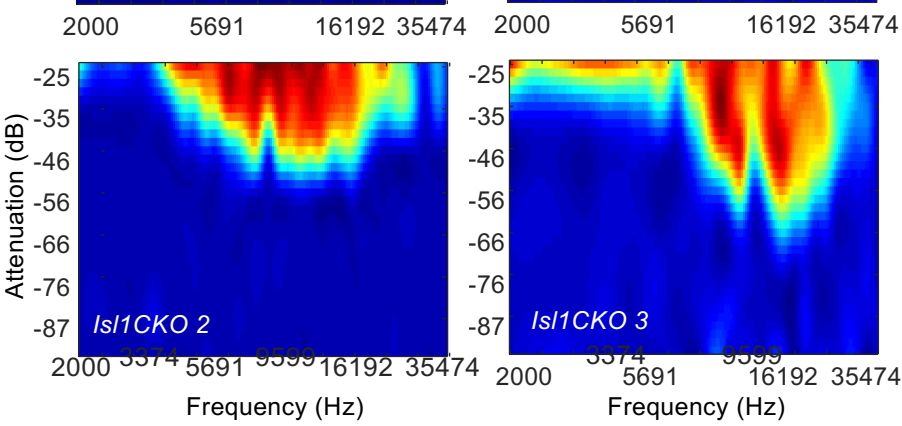
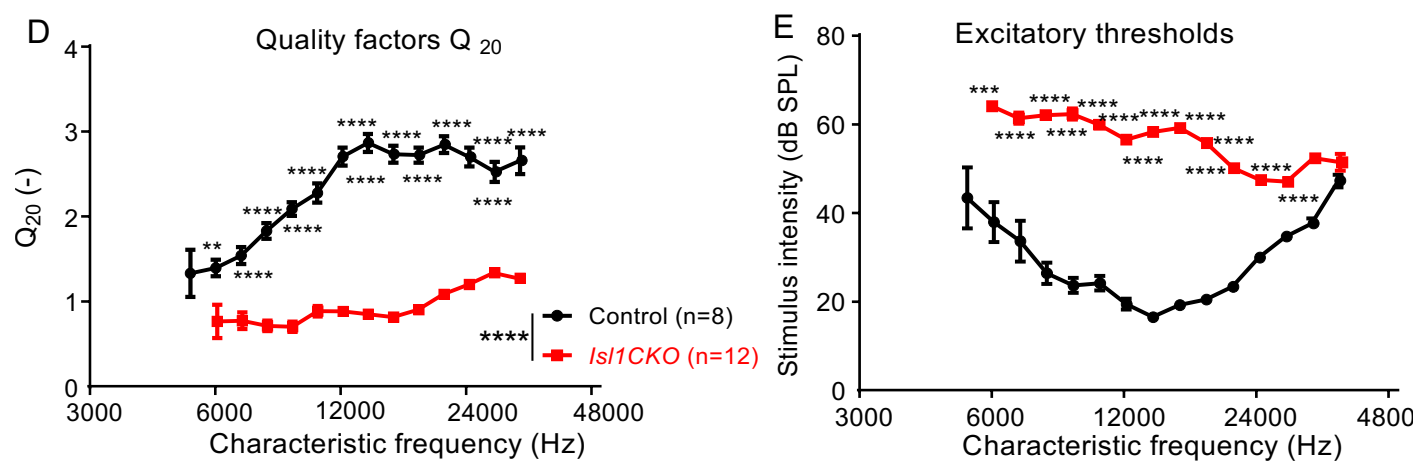

$\mathrm{F}$

BBN threshold

Dynamic range

Spontaneous activity

Max response
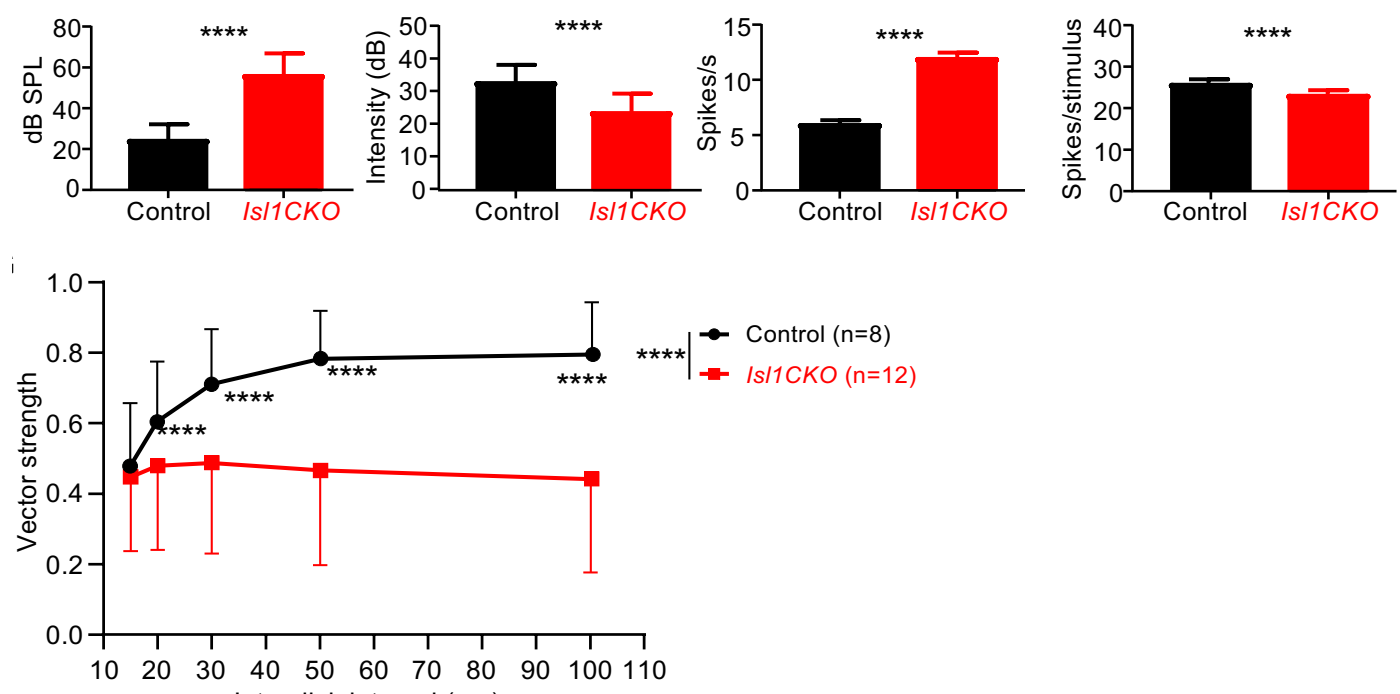

Figure 6. The characteristics of inferior colliculus neurons (units) are affected in Isl1CKO mice. (A) Immunostaining of coronal brain sections for NeuN and HS, nuclear staining Hoechst, and (B) quantification of the volume of the adult control and Isl1CKO inferior colliculus (IC; $\mathrm{n}=5$ ), adjusted to body weight. Data are the mean $\pm \mathrm{SD}$. Two-tailed unpaired t-test (ns, not significant). Scale bars, $200 \mu \mathrm{m}$. HS, nuclear staining Hoechst (blue). (C) Representative examples of tuning curves recorded in the IC display impairments in tuning properties with broad and irregular receptive fields in IsllCKO compared to control mice. (D) Excitatory thresholds of the IC neurons at different characteristic frequencies (CF) are shown as averages in 0.3-octave bins in control and IsllCKO mice. (E) The sharpness of the neuronal tuning expressed by quality factor $\mathrm{Q}_{20}$ (the ratio between the $\mathrm{CF}$ and bandwidth at $20 \mathrm{~dB}$ above the minimum threshold) averaged in 0.3-octave bins is decreased in IsllCKO. Data are mean \pm SEM. Two-way ANOVA with Bonferroni post hoc test. ${ }^{* * \mathrm{P}}<0.01, * * * \mathrm{P}<0.001$, $* * * * \mathrm{P}<0.0001$. (F) Comparison of the rated intensity function parameters between control $(\mathrm{n}=8)$ and IsllCKO $(\mathrm{n}=12)$ mice: broadband noise (BBN) threshold, dynamic range, spontaneous activity, and maximum response magnitude. Data are mean $\pm \mathrm{SD}$; unpaired $t$-test, ${ }^{* * * *} \mathrm{P}<0.0001$. (G) Synchronization of units with click trains. Vector strength computed for different inter-click intervals. Data are the mean $\pm \mathrm{SD}$, two-way ANOVA with Bonferroni post-hoc test, $* * * * \mathrm{P}<0.0001$. 
The tuning properties and excitability of inferior colliculus neurons are distorted in IsllCKO

Having established neuroanatomical changes in the $\mathrm{CN}$, we next evaluated the inferior colliculus (IC) properties, which is the principal auditory structure of the midbrain for the ascending auditory pathways and descending inputs from the ascending auditory pathways auditory cortex ${ }^{42}$. The IC allows for sound localization, integrates multisensory and nonauditory contributions to hearing, and plays an essential role in generating the startle response. We demonstrated no significant IC size reduction in Isl1CKO compared to control mice (Fig. $6 \mathrm{~A}, \mathrm{~B})$. We compared neuronal characteristics in the central nucleus of the IC of Isl1CKO and control animals using multichannel electrodes. Extracellular electrophysiological recordings of neuronal activity in controls showed a well-defined narrow single-peaked profile of the excitatory receptive fields. In contrast to primarily wide-receptive fields, we observed two or more peaks in IsllCKO mice (Fig. 6C), suggesting multiple inputs from the lower levels of the auditory system. A commonly used metric unit of auditory tuning is the "quality factor," or Q, defined as the characteristic frequency (CF) divided by the bandwidth, measured at $20 \mathrm{~dB}$ above the minimum threshold $\left(\mathrm{Q}_{20}\right)$. Results revealed a significantly lower quality factor in the mutant mice (Fig. 6D), showing substantially worsened frequency selectivity.

The investigation of the responsiveness of IC units to different sound frequencies revealed higher excitatory thresholds in IsllCKO than in control animals in all measured frequencies except for the highest recorded frequencies above $28 \mathrm{kHz}$ (Fig. 6E). These comparable excitatory thresholds for high frequencies between control and Isl1CKO mice are consistent with the ABR measurements. We quantified the activity of IC neurons based on the sound intensity, the neuronal responses to a variable intensity of broadband noise (BBN) bursts were analyzed. Compared to control mice, the IC neuronal responses in Isl1CKO had a higher BBN threshold, narrower dynamic range, higher spontaneous activity, and significantly lower maximum response magnitudes (Fig. 6F). The results suggest a functional reduction in sensitivity to sound, audibility, and intensity discrimination, as well as increased excitability of IC neurons in IsllCKO mice.

To evaluate the precise temporal representation of sound into the central auditory system, we performed an acoustic stimulation of the IC units with trains of five clicks with different inter-click intervals from $100 \mathrm{~ms}$ up to $15 \mathrm{~ms}$. In control mice, the increasing time interval between the clicks led to a better synchronization of neuronal responses with the individual clicks in the train, implying the precision and reliability of the temporal sound discrimination ability (Fig. 6G). In contrast, the precise temporal decoding in Isl1CKO was disrupted, as the synchronization of neuronal responses was significantly lower for the whole range of inter-click intervals. In the case of the IsllCKO, the synchronization level of neuronal responses remains almost constant, suggesting a lack of precise temporal sound processing.

\section{The abnormal development of primary auditory neurons alters the auditory behavior of IsllCKO mice}

360 Next, we evaluated the behavioral responses of Isl1CKO mice to sound stimuli. The acoustic 361 startle response (ASR) is usually used as a behavioral readout of hearing status mediated by a brainstem circuit linking cochlear root neurons to spinal motoneurons. The structural basis of the ASR includes cochlear root neurons, neurons of the $\mathrm{CN}$, the nucleus of the lateral lemniscus, the caudal pontine reticular nucleus, spinal interneurons, and spinal motor neurons $43,44,45$. Similar to the ABR thresholds, the ASR thresholds of Isl1CKO significantly increased for startle tone stimuli of $8 \mathrm{kHz}$ and BBN, but not for the high-frequency startle tones (Fig. 7A). The peak latency of the ASR to the BBN stimulation at the $110 \mathrm{~dB}$ SPL intensity was prolonged in IsllCKO compared to control mice (Fig. 7B), indicating a slower reaction to the acoustic stimuli. We found significantly reduced ASR amplitudes for all tested sound stimuli 
A ASR thresholds

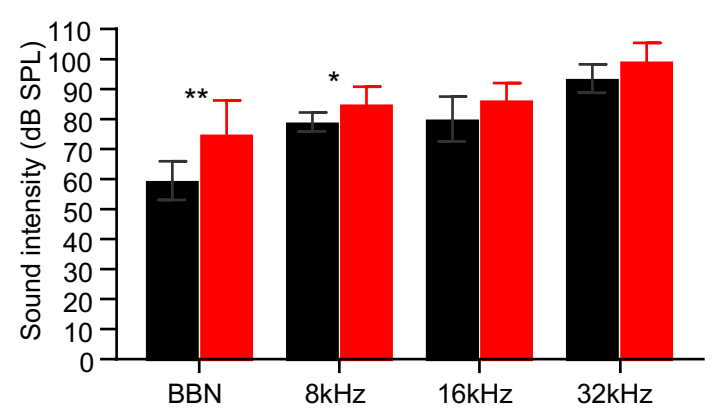

C

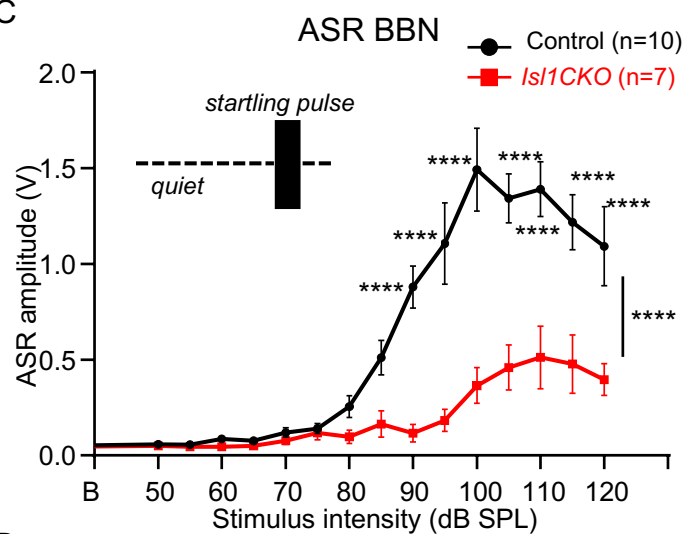

D
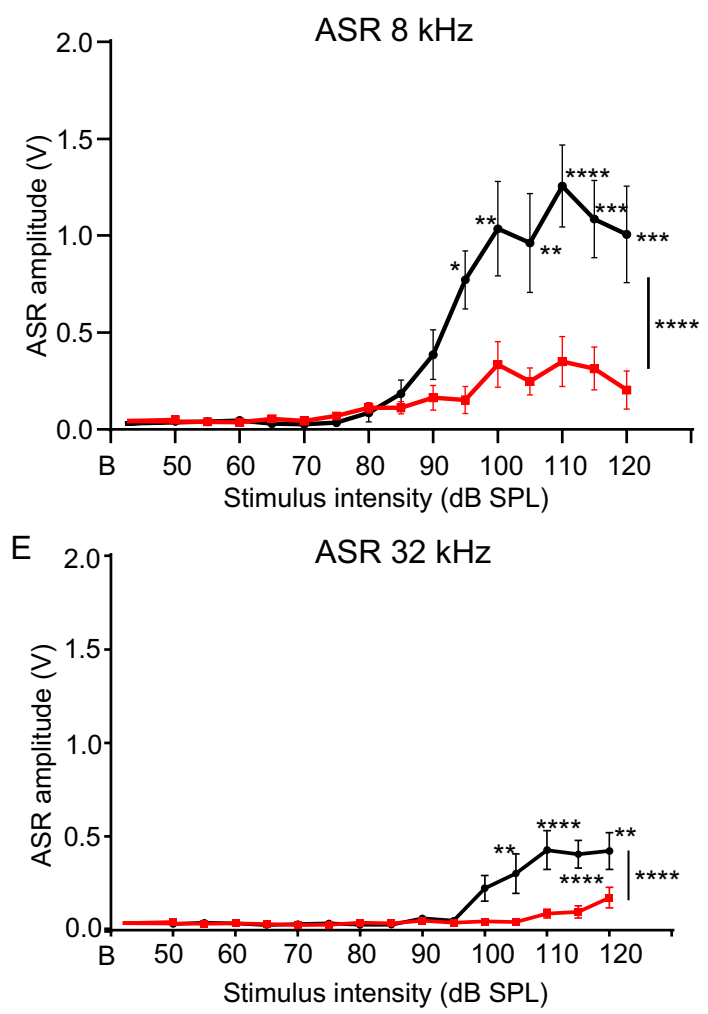

B

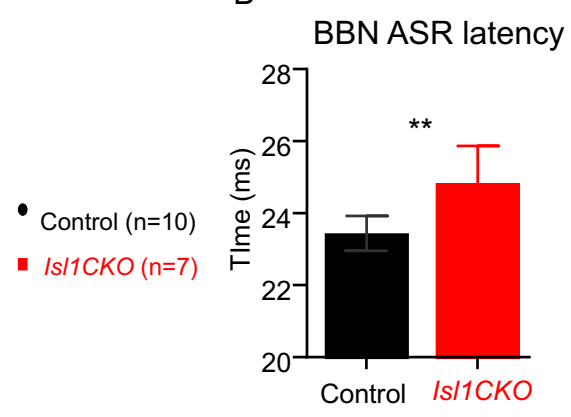

$\mathrm{F}$

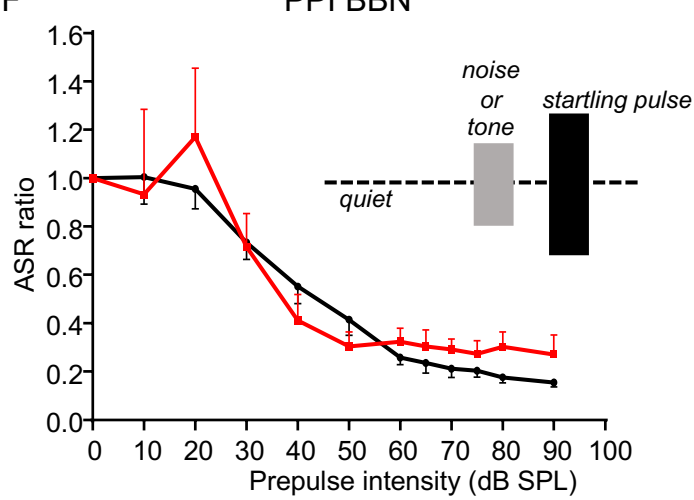

G
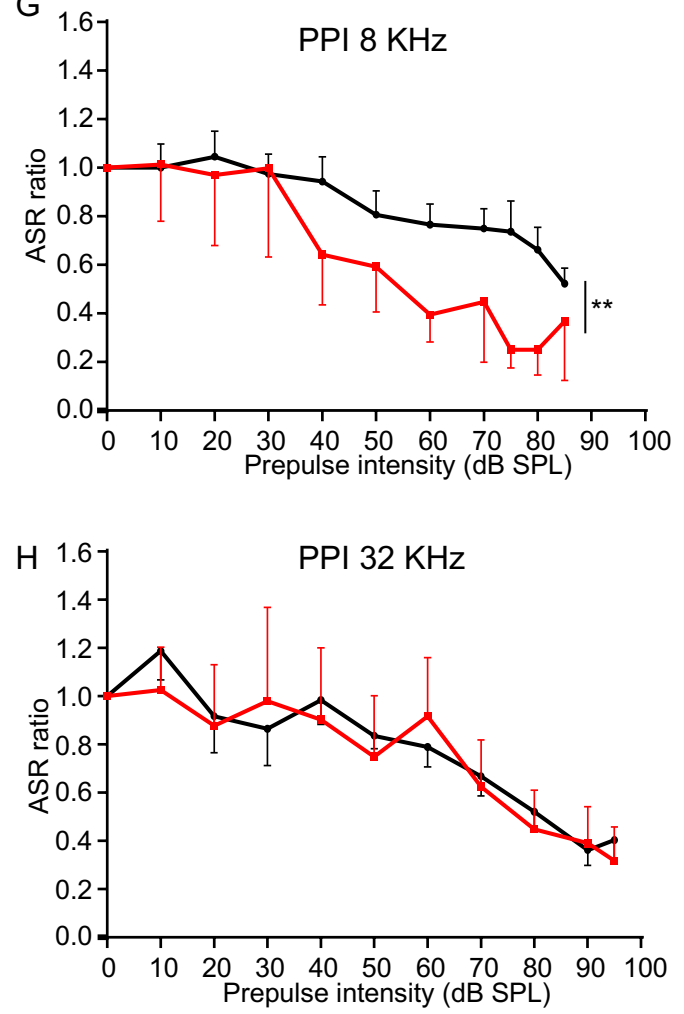

Figure 7. The acoustic startle reflex (ASR) and prepulse inhibition (PPI) responses are altered in the Isl1CKO mutant. (A) The graph shows the ASR thresholds for broadband noise (BBN) bursts and tone pips at 8,16 , and $32 \mathrm{kHz}$ in control and IsllCKO mice. Data are mean \pm SEM. Holm-Sidak method multiple comparison $t$-tests. ${ }^{*} \mathrm{P}<0.05, * * \mathrm{P}<0.01$. (B) Significantly increased ASR latency to BBN is found in IsllCKO compared to control mice. Data are mean $\pm \mathrm{SEM}$. unpaired $t$-test, $* * \mathrm{P}<0.01$. (C) Amplitude-intensity ASR functions for BBN stimulation and (D) for tone pips of $8 \mathrm{kHz}$ and (E) $32 \mathrm{kHz}$ at different dB SPL intensities in control and IsllCKO mice. (F) Efficacy of prepulse intensity on the relative ASR amplitudes was measured the BBN, $(\mathbf{G}) 8 \mathrm{kHz}$, and $(\mathbf{H}) 32 \mathrm{kHz}$ tone prepulse. ASR ratio $=1$ corresponds to the ASR amplitude without a prepulse (the uninhibited ASR). Data are mean $\pm \mathrm{SEM}$. Two-way ANOVA with Bonferroni post hoc tests. $* \mathrm{P}<0.05, * * \mathrm{P}<0.01, * * * \mathrm{P}<0.001, * * * * \mathrm{P}<0.0001$ 
at higher intensities, showing deteriorated acoustic startle reactivity in $I$ sl $1 \mathrm{CKO}$ mice (Fig. 7CE).

To further assess complex auditory discrimination behavior, we exposed control and IsllCKO adult mice to a prepulse inhibition (PPI) paradigm, i.e., the inhibition of the ASR
induced by presenting an acoustic stimulus shortly preceding the presentation of an acoustic induced by presenting an acoustic stimulus shortly preceding the presentation of an acoustic stimulus, the startling sound. The circuit mediating a prepulse on the startle reflex involves central structures of the auditory pathway, including the IC and the auditory cortex ${ }^{46,47}$. We used either BBN or pure tone pips of 8 and $32 \mathrm{kHz}$ at increasing intensities as a non-startling acoustic stimulus (prepulse) that preceded the startle stimulus in a quiet background. The PPI with the prepulse of a pure tone of $32 \mathrm{kHz}$, which is the well-preserved audible frequency in IsllCKO, was comparable between control and mutant mice (Fig. $7 \mathrm{H}$ ). Interestingly, the prepulse with the pure tone of $8 \mathrm{kHz}$ resulted in a larger inhibition of the startle response in IsllCKO than in controls (Fig. 7G), despite the significant hearing deficiency at $8 \mathrm{kHz}$, as shown by ABR evaluations (Fig. 4). This indicates that the $8-\mathrm{kHz}$ prepulse response was enhanced in IsllCKO mice, suggesting compensatory neural hyperactivity of the central auditory system ${ }^{37,48}$. Thus, the ASR and PPI of startle analyses indicate abnormalities of the acoustic behavior of IsllCKO mutants.

\section{Discussion}

Our study shows for the first time that a LIM homeodomain transcription factor ISL1 regulates neuronal development in the cochlea. Using RNA profiling, morphological, and physiological analyses, we provide evidence that ISL1 coordinates genetic networks affecting molecular characteristics of SGNs, their pathfinding abilities, and auditory information processing. The elimination of Isll in neurons during inner ear development results in migration defect of SGNs, disorganized innervation in the cochlea, unsegregated and reduced central axons, and reduced size of the $\mathrm{CN}$. This neuronal phenotype of IsllCKO was accompanied by hearing impairment, abnormalities in sound processing in the IC, and aberrant auditory behavior.

ISL1 is critical for developing multiple tissues, neuronal and non-neuronal cells 49,50 , $51,52,53$. Different aspects of neuronal development depend on ISL1, including specification of motoneurons ${ }^{49}$, sensory neurons ${ }^{22,51}$, axonal growth ${ }^{54}$, and axonal pathfinding ${ }^{55}$. During inner ear development, ISL1 is expressed in both neuronal and sensory precursors ${ }^{14,16,17,18,56}$. Transgenic modulations of Isll expression indicate important roles of ISL1 in the maintenance and function of neurons and hair cells and as a possible contributing factor in neurodegeneration $19,57,58,59$. All these studies suggest that ISL1 plays a role in developing neurons and sensory cells, but no direct evaluation of ISL1 function has been performed. To circumvent the pleiotropic effects of Isll in embryonic development, in this study, we used Neurod1 $1^{\text {Cre }}$ to delete Isll specifically in the inner ear neurons without affecting the development of sensory cells.

We established that ISL1 is necessary for neuronal differentiation programs in the cochlea and the functional properties of the auditory system. Our RNA profiling of SGNs demonstrated transcriptome changes induced by a loss of Isll affecting molecular characteristics of neurons and pathfinding abilities, including neurotransmission, structure of synapses, neuron migration, axonogenesis, and expression of crucial guidance molecules (neurotrophic tyrosine kinase receptors, Ntrk2 and Ntrk3). Consistent with a central role of ISL1 in sensory neuron developmental programs ${ }^{22}$, regulatory networks of signaling molecules and transcription factors were affected in IsllCKO neurons, such as proneural bHLH factors (members of NeuroD, Olig, and Nscl families), LIM-only (Lmo2, Lmo3) and LIM homeodomain transcription factors ( $L h x 1, L h x 2$, Isl2), transcription activation complexes for coordination of particular differentiation programs represented by Eyes absent (Eya4 and Eya2) and Sine oculis (Six2) proteins, Pou $3 f 2$ and Pou $4 f 2$ of the family of POU homeodomain 
trans-regulatory factors, and FGF signaling molecules (Fgflo, Fgfl1, Fgf13, Fgf14) and their downstream Etv transcription factor targets (Etv1, Etv4, Etv5). Interestingly, transcription factor Gata3 was downregulated, suggesting that ISL1 is upstream of the Gata3 transcriptional network differentiation program ${ }^{10}$. Thus, ISL1 orchestrates a complex gene regulatory network driving multiple aspects of differentiation of neurons in the cochlea and defining neuronal features.

The most striking morphological features of the neuronal phenotype of Isl1CKO are the dysregulated migration and pathfinding of SGNs. A similar migration deficit was reported for ErbB2 null mutants ${ }^{60}$; however, the interpretation of findings is compounded by direct inner ear effects and effects associated with neural crest-derived Schwann cells. Additionally, conditional deletion of Sox 10 produced by $W n t 1^{\text {Cre }}$ resulted in abnormal migration of SGNs similar to the IsllCKO phenotype ${ }^{61}$. Migration defects of SGNs in both ErbB2 ${ }^{60}$ and Sox 10 mutants ${ }^{61}$ were attributed to the complete absence of Schwann cells in the entire inner ear ganglion. However, in our IsllCKO mutant, SOX10 positive Schwann cells were found in a similar density in the spiral ganglion of both mutant and control mice (Additional File Fig. $\mathrm{S} 2 \mathrm{C}^{\prime}, \mathrm{D}^{\prime}$ ), thus excluding any direct involvement of glial cells in the migration defects of Islldeficient neurons. Curiously, more profound disorganization of peripheral processes in the cochlea than defects found in Isl1CKO or both ErbB2 null ${ }^{60}$ and Sox 10 mutants ${ }^{61}$ was reported for delayed conditional deletion of Gata3 in SGNs ${ }^{10}$. Despite severe disorganization of cochlear wiring of the Gata3 conditional deletion mutant, central projections maintained their overall tonotopic organization within the auditory nerve and the $\mathrm{CN}^{9}, 10$. In contrast, we provide compelling evidence that elimination of Isll in SGNs affected the pathfinding abilities of neurons in the cochlea not only to form peripheral processes but also to establish central projections. Such profound disorganization of peripheral and central projections is known for Neurod1 mutations ${ }^{13,14,15}$. Deletions of Neurod 1 result in miswired, and reduced SGNs, and loss of tonotopy ${ }^{13,15}$. Somewhat similar disorganization of central axons with unsegregated auditory nerve fibers, reduced the size of the $\mathrm{CN}$, and missing tonotopic organization of synapsing branches in the $\mathrm{CN}$ subdivisions was found in our IsllCKO. Although both Neurod $1 C K O$ and Isl $1 C K O$ demonstrated a significant hearing loss, in contrast to the reduced sound frequency range of Neurod $1 C K \mathrm{O}^{15}$, responses for the entire measured frequency range were detected in IsllCKO. The processing of high acoustic frequencies was broadly comparable between age-matched controls and IsllCKO, indicating some preservation of peripheral neuronal activity. Accordingly, these most preserved high-frequency basal responses correspond to the most close-to-normal distribution of sensory neurons in the cochlear base in IsllCKO mice (Fig. 1).

Nevertheless, as a result of disorganized primary auditory neurons with derailed central projections, the characteristics of persistent auditory function in the IC were altered with worsened tuning capabilities of IC units and their increased spontaneous activity and threshold elevations and decreased dynamic range. The peripheral deficit in sound encoding results in abnormal auditory behavior of $I s l 1 C K O$. Although no significant differences of ABR thresholds at $32 \mathrm{kHz}$ were observed between IsllCKO and control mice, indicating retained hearing function, the startle reactions of $I s l l C K O$ at $32 \mathrm{kHz}$ were reduced. Plasticity of the startle response is also evident in the PPI responses of IsllCKO mice, in which a weak prestimulus suppresses the response to a subsequent startling stimulus. Isl1CKO mice demonstrated PPI impairment for the pure tone of $8 \mathrm{kHz}$, reflecting abnormal sensorimotor gating due to compensatory hyperactivity of the central auditory system ${ }^{48,62}$.

Additionally, compared to control mice, DPOAE responses of Isl1CKO were reduced, indicating dysfunction of cochlear amplification. The OHCs of the organ of Corti play a central role in the active enhancement of sound-induced vibration. For a given OHCs, amplification only occurs at a precise frequency, and thus, this mechanism provides a sharpening of the 
tuning curve and improves frequency selectivity ${ }^{63}$. Nevertheless, DPOAE analysis showed that some function was preserved in high-frequency $\mathrm{OHCs}$ in the IsllCKO cochlea. Frequencies above $28 \mathrm{kHz}$ are located at the basal half of the mouse cochlea from the mid-base to the basal end ${ }^{35}$, which correspond to the most preserved distribution of sensory neurons in the area of the spiral ganglion in the cochlear base of IsllCKO mice (Fig. 1H). Usually, decreased DPOAE amplitudes indicate loss and dysfunction of OHCs 57,63, 64, 65. Since Neurod $1^{\mathrm{Cre}}$ is not expressed in sensory cells in the cochlea, it is unlikely that the development of OHCs is directly affected in IsllCKO. Instead, cochlear amplification deficits in IsllCKO correlated with the reduced and disorganized innervation of OHCs, as shown in Fig. 1. The medial olivocochlear efferents innervate $\mathrm{OHCs}$ from the brainstem, representing a soundevoked negative feedback loop suppressing $\mathrm{OHC}$ activity ${ }^{66}$. Besides efferents, OHCs are innervated by the type II SGNs ${ }^{67,68}$. Although the function of type II SGNs remains obscure, it is clear that these neurons are involved in auditory nociception ${ }^{69}$, and may also constitute the sensory drive for the olivocochlear efferent reflex ${ }^{70}$ that is disputed ${ }^{71}$. As $I s l 1$ is expressed in both type I and type II SGNs during inner ear development ${ }^{20}$, characteristics of both neuronal types might likely be affected in IsllCKO.

An additional morphological change likely contributing to the hearing deficit of IsllCKO is a shortened cochlea. Somewhat similar phenotypes of a shortened cochlea were previously reported for deletion mutants of Neurod 1 and Neurogl, key transcription factors for inner ear neuronal development ${ }^{8,14,15,72}$. A comparable effect of cochlear length reduction was observed following the loss of $\mathrm{Foxg}{ }^{73}$ and $\operatorname{Lmxla} \mathrm{a}^{74,75}$. Although mechanisms affecting the cochlear extension are unknown, it is clear that this confounding feature of the Isl1CKO phenotype would consequently impact mechanical and neural tuning from the base to the apex of the cochlea and the ability to perform time-frequency processing of sound.

\section{Conclusions}

Our study provides compelling evidence that ISL1 is a critical regulator of SGN development, affecting neuronal migration, pathfinding abilities to form cochlear wiring, and central axonal projections. As such, ISL1 represents an essential factor in the regulation of neuronal differentiation to produce functional neurons in cell-based therapies and stem cell engineering 76,77. Additionally, this unique model contributes to our understanding of how disorganization of the neuronal periphery affects information processing at higher centers of the central auditory pathway at the physiological and behavioral levels. ISL1 is a LIM-homeodomain transcription factor with a specific potential to interact and recruit other co-factors to form higher-order regulatory complexes ${ }^{78}$. In the future, it will be intriguing to fully identify the molecular mechanisms and co-regulators underlying ISL1 function in SGNs.

\section{Methods}

\section{Experimental animals}

All methods were performed according to the Guide for the Care and Use of Laboratory Animals (National Research Council. Washington, DC. The National Academies Press, 1996). The design of experiments was approved by the Animal Care and Use Committee of the Institute of Molecular Genetics, Czech Academy of Sciences. The mice were housed in 12hour light/dark cycles and were fed ad libitum. To generate IsllCKO (the genotype Neurod1 ${ }^{\text {Cre }} ;$ Isl $11^{\text {loxP/loxP }}$ ), we cross-bred floxed Isl11 (Isl ${ }^{\text {loxP/loxP }} ;$ Isl1 $1^{\text {tm } 2 S e v} / J$, \# 028501, Jackson

Laboratory) ${ }^{22}$ and Neurod1 ${ }^{\text {Cre }}$ transgenic mice (Tg(Neurod1-cre)1Able/J, \# 028364, Jackson

518 Laboratory), which were generated by pronuclear injection of the Neurodl-cre BAC construct

519 that carries Cre-sequence downstream of the translational initiation codon ATG of the Neurod1 
521 Mendelian ratios, and were phenotypically indistinguishable from control (Cre negative)

522 littermate mice. As control mice, we used mice with the genotype Cre negative, Is $l 1^{\text {loxPloxP }}$ and 523 Isl $11^{+/ l o x P}$. The mouse line Neurodl ${ }^{\text {Cre }}$ was also bred with Cre-reporter tdTomato line

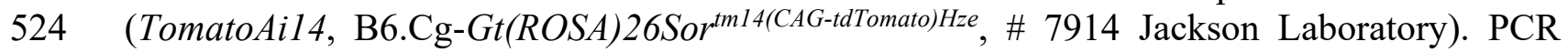
525 performed genotyping on tail DNA. We used both males and females for experiments. Lines 526 are a mixed C57BL/6/sv129 background. Phenotyping and data analysis was performed blind 527 to the genotype of the mice.

\section{Morphological evaluation of the cochlea, cochlear nucleus, and inferior colliculus}

Dissected ears were fixed in 4\% paraformaldehyde (PFA) in PBS. For vibratome sections, samples were embedded in $4 \%$ agarose and sectioned at $80 \mu \mathrm{m}$ using a Leica VT1000S vibratome. Vibratome sections, whole inner ears, or whole embryos were defatted in $70 \%$ ethanol and then rehydrated and blocked with serum, as described previously ${ }^{16,58}$. Samples were then incubated with primary antibodies at $4^{\circ} \mathrm{C}$ for 72 hours. The primary antibodies used were: rabbit anti-Myosin 7a (Myo7a; Proteus BioSciences 25-6790, 1:500), mouse antiacetylated $\alpha$-tubulin (tubulin; Sigma-Aldrich T6793, 1:400), rabbit anti-calretinin (Santa Cruz Biotechnology sc-50453, 1:100), rabbit anti-parvalbumin (Abcam ab11427, 1:2000), mouse anti-VGLUT1 (Merck MAB5502, 1:200), rabbit anti-NeuN (Abcam ab177487, 1:500), goat anti-prestin (Santa Cruz Biotechnology sc-22692, 1:50), mouse anti-Isl1 (Developmental Hybridoma Bank 39.3F7, 1:130), goat anti-Neurod1 (Santa Cruz Biotechnology sc-1084, 1:100), and rabbit anti-Sox10 (Abcam ab155279, 1:250). After several PBS washes, secondary antibodies were added and incubated at $4^{\circ} \mathrm{C}$ for 24 hours. The secondary antibodies Alexa Fluor ${ }^{\circledR} 488$ AffiniPure Goat Anti-Mouse IgG (Jackson ImmunoResearch Laboratories 115545-146), Alexa Fluor ${ }^{\circledR} 594$ AffiniPure Goat Anti-Rabbit IgG (Jackson ImmunoResearch Laboratories 111-585-144), and DyLight488-conjugated AffiniPure Mouse Anti-Goat IgG (Jackson ImmunoResearch Laboratories 205-485-108) were used in 1:400 dilution. Nuclei were stained by Hoechst 33258 (Sigma-Aldrich 861405, 1:2000). Samples were mounted in Aqua-Poly/Mount (Polysciences 18606) or prepared Antifade medium, and images were taken on Zeiss LSM 880 confocal microscope. ImageJ and ZEN software was used for image processing.

The length of the organ of Corti was measured using the "Measure line" ImageJ plugin. The $\mathrm{CN}$ and inferior colliculus volumes were established by analyzing parallel, serial equally spaced $80 \mu \mathrm{m}$ coronal vibratome sections through the brain $(\mathrm{n}=5$ IsllCKO and $\mathrm{n}=5$ control mice). The left and right cochlear nucleus and inferior colliculus areas were determined in each section using ImageJ, and the volume of the organs was calculated. Volumes of paired organs were adjusted to the body weight. Whole-mount anti-tubulin labeling of the cochlea was used to measure the length and density of the radial fibers. The evaluation of the innervation was done separately for each part of the cochlea: the apex, mid-apex, mid-base, and base. Due to the disorganization of innervation in the apex, we only evaluated the fiber density in the cochlea's base, mid-base, and mid-apex parts. The density of the radial fibers was expressed as the percentage of a positive area in the measured area of $152 \times 66 \mu \mathrm{m}^{2}$ using the "Threshold" function ImageJ. The length of the radial fibers was measured from the whole-mount antitubulin and Myo7a immunolabeled cochlea confocal images. For each genotype, we measured 3 samples, and in each part of the cochlea, we measured the length of the fibers in 3 radial fiber bundles from the IHCs to the IGSB. To compare how many neurons are correctly located in the area of the Rosental's canal in IsllCKO, we used NeuN immunolabeled whole-mount cochlea. We measured the corresponding region of the spiral ganglion-containing neurons using the "Threshold" function ImageJ. We used 5 samples per genotype.

\section{Isolation of genetically labeled neurons and library construction}


Spiral ganglia were micro-dissected in Dulbecco's PBS on ice from E14.5 embryos of either sex from four litters; spiral ganglia from both inner ears of the individual embryo per sample. Spiral ganglia were incubated in $300 \mu \mathrm{l}$ of lysis solution $(0.05 \%$ trypsin, $0.53 \mathrm{mM}$ EDTA Dulbecco's PBS) in $37^{\circ} \mathrm{C}$, shaking at $900 \mathrm{RPM}$ for $5 \mathrm{~min}$. The lysis was stopped by adding $600 \mu 1$ of FACS buffer (Dulbecco's PBS, 10mM EGTA, and 2\% FBS). After spinning down the samples at $800 \mathrm{G}, 4{ }^{\circ} \mathrm{C}$ for $10 \mathrm{~min}$, the supernatant was removed, and cell pellets were resuspended in $500 \mu \mathrm{l}$ of ice-cold FACS buffer. Immediately before sorting, cells were passed through a $50 \mu \mathrm{m}$ cell sieve (CellTrics ${ }^{\mathrm{TM}}$, Sysmex Amercica Inc.) into a sterile $5 \mathrm{ml}$ polystyrene round-bottom falcon to remove clusters of cells and kept on ice. TdTomato ${ }^{+}$ neurons were sorted using a flow cytometer (BD FACSAria ${ }^{\mathrm{TM}}$ Fusion), through a $100 \mu \mathrm{m}$ nozzle in 20 psi, operated with BD FACSDiva ${ }^{\mathrm{TM}}$ Software. 100 sorted cells were collected into individual wells of 96-well plate containing $5 \mu 1$ of lysis buffer of NEB Next single-cell low input RNA library prep kit for Illumina (\#E6420 New England Biolabs). Plates were frozen immediately on dry ice and stored at $-80^{\circ} \mathrm{C}$. The total time from euthanasia to cell collection was $\sim 3$ hrs.

RNAseq-libraries were prepared from 6 samples per each genotype, control (Neurod ${ }^{\text {Cre }}$; TomatoAi14) and Isl1CKO mutant (IsllCKO;TomatoAi14), and each sample contained 100 tdTomato $^{+}$neurons. Following the manufacturer's instructions, the NEB Next single-cell low input RNA library prep kit for Illumina was used for cDNA synthesis, amplification, and library generation ${ }^{79}$ at the Gene Core Facility (Institute of Biotechnology CAS, Czechia). Fragment Analyzer assessed the quality of cDNA libraries. The libraries were sequenced on an Illumina NextSeq 500 next-generation sequencer. NextSeq 500/550 High Output kit 75 cycles (\# 200024906, Illumina) were processed at the Genomics and Bioinformatics Core Facility (Institute of Molecular Genetics CAS, Czechia).

\section{Computational analysis of RNAseq data}

RNA-Seq reads in FASTQ files were mapped to the mouse genome using STAR [version 2.7.0 ${ }^{80}$ ] version GRCm 38 primary assembly and annotation version M8. The raw data of RNA sequencing were processed with a standard pipeline. Using cutadapt v1.18 ${ }^{81}$, the number of reads (minimum, 32 million; maximum, 73 million) was trimmed by Illumina sequencing adaptor and of bases with read quality lower than 20 , subsequently reads shorter than $20 \mathrm{bp}$ were filtered out. TrimmomaticPE version $0.36^{82}$. Ribosomal RNA and reads mapping to UniVec database were filtered out using bowtie v1.2.2. with parameters $-\mathrm{S}-\mathrm{n} 1$ and SortMeRNA ${ }^{83}$. A count table was generated by Rsubread v2.0.1 package using default parameters without counting multimapping reads. The raw RNAseq data were deposited at GEO: \# GSE182575 study (https://www.ncbi.nlm.nih.gov/geo/query/acc.cgi?acc=GSE182575). DESeq2 [v1.26.0 $\left.{ }^{84}\right]$ default parameters were used to normalize data and compare the different groups. Differentially expressed genes were identified based on an adjusted P-value $\mathrm{p}_{\mathrm{adj}}<0.05, \mathrm{FC}>2$, and a base mean $\geq 50$ was applied to identify differentially expressed genes between IsllCKO mutant and control neurons. The functional annotation of the differentially expressed genes was performed using GOTermFinder ${ }^{85}$.

Enrichment mapping. The enrichment of the functional categories and functional annotation clustering of the differentially expressed genes were performed using g: Profiler ${ }^{86}$ input using version e104_eg51_p15_3922dba with g: SCS multiple testing correction methods applying significance threshold of 0.05 , while no electronic GO annotations were used. Only Biological Processes (BP) data underwent further processing. Complete query details are available in Query info tabs in Additional Supplementary file 2, Table S2, S3. The resulting GEM and combined GMT files were loaded into Cytoscape ${ }^{87}$ plugin "EnrichmentMap" 88 using 0.01 FDR q-value cutoff to generate a network. Edge cutoff was set to 0.6 , and nodes were filtered by gs_size $<1800$. Five GO terms forming solitary nodes, or a pair of nodes, were excluded 
621 (listed in Additional Supplementary file 2, Table S2, S3). Further adjustments were made in 622 yFiles Layout Algorithms, Legend Creator (Cytoscape plugins), and Inkscape (Inkscape 623 Project, 2020).

\section{Quantitative real-time PCR}

626 Total RNA was isolated from both inner ears of the embryo at E14.5 using TRI Reagent 627 (Sigma-Aldrich T9424). We used 8 embryos for IsllCKO and 7 embryos for the control group 628 from three litters. RNA from both inner ears of one embryo represented one sample. RNA 629 samples $(1 \mu \mathrm{g})$ were processed and analyzed as previously described ${ }^{58}$. Briefly, following RT, 630 quantitative qPCR was performed with initial activation at $95{ }^{\circ} \mathrm{C}$ for $120 \mathrm{~s}$, followed by 40 631 cycles at $95{ }^{\circ} \mathrm{C}$ for $15 \mathrm{~s}, 60{ }^{\circ} \mathrm{C}$ for $30 \mathrm{~s}$, and $72{ }^{\circ} \mathrm{C}$ for $30 \mathrm{~s}$ using the CFX384 ${ }^{\mathrm{TM}}$ Real-Time

632 PCR Detection System (Bio-Rad Laboratories). The primer sequences 633 (pga.mgh.harvard.edu/primerbank/) are listed in Additional Supplementary file 2, Table S4. 634 Relative mRNA expression was calculated using the $-\Delta \Delta \mathrm{Cq}$ method with Hprt1 as a reference gene. GraphPad Prism software was used for statistical analysis.

\section{Lipophilic Dye Tracing}

We studied the innervation pattern in whole or dissected ears using lipophilic dye tracing in aldehyde-fixed tissues as previously described ${ }^{89}$. At least three mutants and similar numbers of control littermates of both sexes were used for each evaluation. Filter strips loaded with colored lipophilic dyes were inserted into the cochlear apex, base, and vestibular end-organ utricle ${ }^{90}$. After allowing appropriate time for diffusion of the lipophilic tracer (between 48120 hours), we prepared the ears as whole mounts in glycerol on a glass slide, using appropriate spacers to avoid distortion, and imaged them using a Leica SP8 confocal microscope. Images were compiled into plates to show the most pertinent details using Corel Draw. Only general image modifications such as contrast or brightness adjustments were used to enhance the visual appeal without affecting the scientific content.

\section{Hearing function evaluation}

Auditory brainstem response (ABR) and distortion product otoacoustic emissions (DPOAEs) tests were carried out on mice under general anesthesia with $35 \mathrm{mg} / \mathrm{kg}$ ketamine (Calypso 50 $\mathrm{mg} / \mathrm{ml}$ ) and $6 \mathrm{mg} / \mathrm{kg}$ xylazine (Xylapan $20 \mathrm{mg} / \mathrm{ml}$ ) in saline to give an application volume of 7 $\mathrm{ml} / \mathrm{kg}$ body weight via subcutaneous injection, maintained on a temperature-regulated blanket in a soundproof room.

Distortion product otoacoustic emissions. For DPOAE recording were tested $I \operatorname{sllCKO}(\mathrm{n}=10)$ and control mice $(\mathrm{n}=14)$. Cubic $(2 \mathrm{~F} 1-\mathrm{F} 2)$ distortion product otoacoustic emissions over an F2 frequency range from 6 to $38 \mathrm{kHz}$ were recorded with a low-noise microphone system (Etymotic probe ER-10B+, Etymotic Research). Acoustic stimuli (ratio F2/F1 = 1.21, F1 and F2 primary tone levels of $\mathrm{L} 1 / \mathrm{L} 2=70 / 60 \mathrm{~dB}$ ) were presented to the ear canal with two custommade piezoelectric stimulators connected to the probe with $10-\mathrm{cm}-$ long silastic tubes. The signal from the microphone was analyzed by the TDT System III (RP2 processor, sampling rate $100 \mathrm{kHz}$ ) using custom-made MATLAB software. DPOAEs were recorded in the animals' ears at individual frequencies over the frequency range $4-38 \mathrm{kHz}$ with a resolution of ten points per octave. All the experiments and analyses were done with no information on the genotype. Auditory brainstem response. For auditory brainstem response (ABR) recording ( $\mathrm{n}=7$ IsllCKO and $\mathrm{n}=10$ control mice), an active electrode was placed subcutaneously on the vertex and ground and reference electrodes in the neck muscles. Responses to tone bursts $(3 \mathrm{~ms}$ duration, $1 \mathrm{~ms}$ rise/fall times, frequencies of 2, 4, 8, 16, 32, and $40 \mathrm{kHz}$ ) and clicks of different intensity were recorded. Acoustic stimuli were conveyed to the animal in free-field conditions via a two-way loudspeaker system (Selenium 6W4P woofer and RAAL70-20 tweeter) placed 
$70 \mathrm{~cm}$ in front of the animal's head. The signal was processed with a TDT System III Pentusa Base Station and analyzed using BioSig ${ }^{\mathrm{TM}}$ software. The response threshold to each frequency was determined as the minimal tone intensity that still evoked a noticeable potential peak in the expected time window of the recorded signal. The amplitude and latency of ABR peaks I$\mathrm{V}$ were determined using BioSig software (Tucker Davis Technologies). Central compensation of neuronal responsiveness (central gain) was calculated using ABR wave IV to I amplitudes.

\section{Extracellular recording of the neuronal activity in the inferior colliculus (IC)}

680 The surgery and extracellular recording in the IC were performed in mice anesthetized with 35 $681 \mathrm{mg} / \mathrm{kg}$ ketamine (Calypsol $50 \mathrm{mg} / \mathrm{ml}$ ) and $6 \mathrm{mg} / \mathrm{kg}$ xylazine (Xylapan $20 \mathrm{mg} / \mathrm{ml}$ ) in saline via 682 subcutaneous injection. Approximately every hour, supplement subcutaneous injections of one-half of the original dose of the anesthetics were administered to keep a sufficient level of anesthesia, judged by a positive pedal and palpebral (toe-pinch) reflex and movement of the whiskers. Respiratory rate, and heart rate, were monitored. An incision was made through the skull's skin for access to the IC, and underlying muscles were retracted to expose the dorsal skull. A holder was glued to the skull, and small holes were drilled over both ICs. Neuronal activity (multiple units) in the IC was recorded using a 16-channel, single shank probe (NeuroNexus Technologies) with 50 or $100 \mu \mathrm{m}$ between the electrode spots. The obtained signal from the electrode was amplified 10000 times, band-pass filtered over the range of 300 $\mathrm{Hz}$ to $10 \mathrm{kHz}$ and processed by a TDT System III (Tucker Davis Technologies) using an RX52 Pentusa Base Station. Individual spikes from the recorded signal were isolated online based on amplitude discrimination and analyzed with BrainWare software (v. 8.12, Jan Schnupp, Oxford University). Subsequent discrimination of spikes from the recorded data and their sorting according to the amplitudes of the first positive and negative peaks were performed offline and was used to sort action potentials (spikes) among single units. The recorded data were processed and analyzed using custom software based on MATLAB. The stimulation signals were generated using a TDT System III with the RP 2.1 Enhanced Real-Time Processor. Acoustic stimuli were delivered in free-field conditions via a two-driver loudspeaker system (Selenium 6W4P woofer and RAAL70-20 tweeter) placed $70 \mathrm{~cm}$ in front of the animal's head. Frequency-intensity mapping: To determine the neuronal receptive fields, pure tones (frequency $2-40 \mathrm{kHz}$ with $1 / 8$ octave step, $60 \mathrm{~ms}$ duration, $5 \mathrm{~ms}$ rise/fall times, various intensity with $5 \mathrm{~dB}$ step) were presented in a random order, each stimulus appearing three times. A discrete matrix corresponding to the response magnitude evoked by each of the frequencyintensity combinations was thereby obtained, smoothed using cubic spline interpolation, and used for extraction of the basic parameters: the excitatory response threshold (the lowest stimulus intensity that excited the neuron, measured in $\mathrm{dB}$ SPL), the characteristic frequency (CF) - the frequency with the minimal response threshold, measured in $\mathrm{Hz}$, and the bandwidth of the excitatory area $20 \mathrm{~dB}$ above the excitatory threshold, expressed by quality factor $\mathrm{Q}(\mathrm{Q}=$ $\mathrm{CF} / \mathrm{bandwidth).}$

Rate intensity function of the IC neurons: Neuronal responses to broadband noise (BBN) bursts of variable intensity ( $10 \mathrm{~dB}$ steps, 50 repetitions) were used to construct the rate intensity function (RIF). A 100\% scale was assigned to the neuron's total range of response amplitudes, $0 \%$ corresponding to spontaneous activity and $100 \%$ corresponding to its maximum response magnitude ${ }^{91}$. The two points of interest are R10 and R90, which correspond to 10 and $90 \%$ of this scale, respectively. R10, describing the starting point of the RIF's rise, was taken as the BBN response threshold. RIFs were further used for evaluating the following parameters: the dynamic range $(\mathrm{DR})$ of the RIF: $\mathrm{DR}=\mathrm{S} 90-\mathrm{S} 10$ ); and the maximum response magnitude. Spontaneous activity of the IC neurons was determined at the OdB SPL BBN stimulation. 
Temporal properties of the IC neurons: We used trains of five clicks at an intensity of $70 \mathrm{~dB}$ SPL for control and $80 \mathrm{~dB}$ SPL for IsllCKO mice with various inter-click intervals $(100,50$, 30,20 , and $15 \mathrm{~ms}$ ). We calculated the vector strength (VS) values and the Rayleigh statistics for each spike pattern; only responses with at least 5.991 were significantly considered phaselocking (Zhou and Merzenich, 2008). The VS quantifies how well the individual spikes are synchronized (phase-locked) with a periodic signal.

\section{Behavioral tests}

IsllCKO $(\mathrm{n}=10)$ and control $(\mathrm{n}=7)$ mice were used at 2-3 months of age. All behavioral tests were performed in a sound-attenuated chamber (Coulbourn Habitest, model E10-21) located in a soundproof room. Each mouse was placed in a wire mesh cage on a motion-sensitive platform inside the box during the testing. The mouse's reflex movements to sound stimuli were detected and transformed to a voltage signal by the load-cell response sensing platform. An amplified voltage signal was acquired and processed using a TDT system 3 with a RealTime Processor RP 2 (Tucker Davis Technologies, Alachua, Fl) and custom-made software in a Matlab environment. The startle responses were evaluated in $100 \mathrm{~ms}$ windows, beginning at the onset of the startling stimulus. The magnitude of the response was measured as the maximal peak-to-peak amplitude of transient voltage occurring in the response window. Acoustic stimuli were generated by the TDT system (Real-Time Processor RP 2), amplified and presented via a loudspeaker (SEAS, 29AF/W), and placed inside the chamber above the animal. Stimulus presentation and data acquisition were controlled by a custom-made application in a Matlab environment. Calibration of the apparatus was performed for frequencies between 4 $\mathrm{kHz}$ and $32 \mathrm{kHz}$ by a 1/4-inch Brüel \& Kjaer 4939 microphone connected to a Brüel \& Kjaer ZC 0020 preamplifier and a B\&K 2231 sound level meter. During the calibration, the calibrating microphone was positioned at the animal's head in the test cage.

Acoustic startle reflex (ASR) (a transient motor response to an intense, unexpected stimulus) was used to indicate the behavioral responsiveness to sound stimuli. The ASRs to 8, 16, and $32 \mathrm{kHz}$ tone pips and BBN bursts ( $50 \mathrm{~ms}$ duration, $3 \mathrm{~ms}$ rise/fall times, varying intensity levels) were recorded. Each test session contained: a baseline trial ( $-10 \mathrm{~dB}$ SPL stimulus intensity) and 13 startle stimuli of different intensities $(50,55,60,65,70,75,80,85,90,100,110,115$, and $120-\mathrm{dB}$ SPL). The inter-trial interval varied from 15 to $50 \mathrm{~s}$.

In the prepulse inhibition (PPI) procedure, 3 different trial types were used: a baseline trial without any stimulus, an acoustic startle pulse alone (white noise at $110 \mathrm{~dB}$ SPL, $50 \mathrm{~ms}, 3 \mathrm{~ms}$ rise/fall times), and a combination of the prepulse and startle pulse. The inter-stimulus interval between the prepulse and the startle stimulus was set to $50 \mathrm{~ms}$; each trial type was presented three times. The inter-trial gap was randomized and varied from 15 to $50 \mathrm{~s}$. The efficacy of the PPI of ASR was expressed as an ASR ratio in percentage, e.g., 100\% corresponds to the amplitude of ASR without prepulse; smaller values of ASR ratio indicate stronger PPI. As a prepulse, we used either BBN bursts or tone pips (50 ms duration, $3 \mathrm{~ms}$ rise/fall time) at frequencies of 8 and $32 \mathrm{kHz}$ at increasing intensities. It is expected that in the presence of prepulse, the amplitude of the following startle response decreases.

\section{Experimental design and statistical analyses}

All comparisons were made between animals with the same genetic background, typically littermates, and we used both male and female mice. The number of samples (n) for each comparison can be found in the individual method descriptions and are given in the corresponding figures. Phenotyping and data analysis was performed blind to the genotype of the mice. All values are presented either as the mean \pm standard deviation (SD) or standard error of the mean (SEM). For statistical analysis, GraphPad Prism software was used. To assess differences in the mean, one-way or two-way ANOVA with Bonferroni's multiple comparison 
test, multiple $t$-tests with Holm-Sidak comparison method, and unpaired two-tailed $t$-tests were employed. Significance was determined as $\mathrm{P}<0.05\left(^{*}\right), \mathrm{P}<0.01(* *), \mathrm{P}<0.001(* * *)$ or $\mathrm{P}<$ $0.0001(* * *)$. Complete results of the statistical analyses are included in the figure legends.

\section{Ethics approval and consent to participate}

Experiments were carried out following the animal welfare guidelines 2010/63/EC of the European Communities Council Directive, agreeing with the Guide for the Care and Use of Laboratory Animals (National Research Council. Washington, DC. The National Academies Press, 1996). The design of experiments was approved by the Animal Care and Use Committee of the Institute of Molecular Genetics, Czech Academy of Sciences (protocol \# 104/2019).

\section{Consent for publication}

Not applicable.

\section{Data availability}

All data generated or analyzed during this study are included in this published article, its Additional Supplementary Files 1, 2, and in the publicly available Gene Expression Omnibus (GEO) repository.

\section{Competing interests}

The authors declare that they have no competing interests.

\section{Funding}

This research was supported by the Czech Science Foundation (20-06927S to GP), by the institutional support of the Czech Academy of Sciences (RVO: 86652036 to GP), and NIH/NIA (R01 AG060504, DC016099, AG051443 to BF and ENY).

\section{Authors' contributions}

GP, JS, BF designed and supervised the experiments. IF, KP, RB, MT, SV, MD, and BF performed experiments and analyzed the data. OS, SB, and LV carried RNAseq analyses. IF and KP prepared the first draft of the manuscript. GP and BF wrote the manuscript, ENY and JS reviewed the manuscript. All authors read and approved the final manuscript.

\section{Acknowledgments}

A. Pavlinek (King's College London) for editing the MS. We acknowledge Imaging Methods Core Facility at BIOCEV supported by the MEYS CR (Large RI Project LM2018129 CzechBioImaging) and ERDF (project No. CZ.02.1.01/0.0/0.0/18_046/0016045) for its support with obtaining imaging data presented in this paper and FAC $\bar{C}$ experiments, and Biocev GeneCore Facility for its support with gene expression/transcriptome analyses.

\section{References}

1. Muniak MA, Connelly CJ, Suthakar K, Milinkeviciute G, Ayeni FE, Ryugo DK. Central Projections of Spiral Ganglion Neurons. In: The Primary Auditory Neurons of the Mammalian Cochlea). Springer (2016).

2. Rubel EW, Fritzsch B. Auditory system development: primary auditory neurons and their targets. Annu Rev Neurosci 25, 51-101 (2002). 
818 3. Kandler K, Clause A, Noh J. Tonotopic reorganization of developing auditory brainstem circuits. Nat Neurosci 12, 711-717 (2009).

820

821

822

823

824

825

826

827

828

829

830

831

832

833

834

835

836

837

838

839

840

841

842

843

844

845

846

847

848

849

850

851

852

853

854

855

856

857

858

859

860

861

4. Di Bonito M, Studer M. Cellular and Molecular Underpinnings of Neuronal Assembly in the Central Auditory System during Mouse Development. Front Neural Circuits 11, 18 (2017).

5. Lopez-Poveda EA. Olivocochlear Efferents in Animals and Humans: From Anatomy to Clinical Relevance. Front Neurol 9, 197 (2018).

6. Fritzsch B, Elliott KL. Evolution and Development of the Inner Ear Efferent System: Transforming a Motor Neuron Population to Connect to the Most Unusual Motor Protein via Ancient Nicotinic Receptors. Front Cell Neurosci 11, 114 (2017).

7. Ma Q, Anderson DJ, Fritzsch B. Neurogenin 1 null mutant ears develop fewer, morphologically normal hair cells in smaller sensory epithelia devoid of innervation. $J$ Assoc Res Otolaryngol 1, 129-143 (2000).

8. Kim W-Y, et al. NeuroD-null mice are deaf due to a severe loss of the inner ear sensory neurons during development. Development 128, 417-426 (2001).

9. Duncan JS, Fritzsch B. Continued expression of GATA3 is necessary for cochlear neurosensory development. PloS one 8, e62046 (2013).

10. Appler JM, Lu CC, Druckenbrod NR, Yu WM, Koundakjian EJ, Goodrich LV. Gata3 is a critical regulator of cochlear wiring. J Neurosci 33, 3679-3691 (2013).

11. Huang EJ, Liu W, Fritzsch B, Bianchi LM, Reichardt LF, Xiang M. Brn3a is a transcriptional regulator of soma size, target field innervation and axon pathfinding of inner ear sensory neurons. Development 128, 2421-2432 (2001).

12. Liu M, et al. Essential role of BETA2/NeuroD1 in development of the vestibular and auditory systems. Genes \& development 14, 2839-2854 (2000).

13. Jahan I, Kersigo J, Pan N, Fritzsch B. Neurod1 regulates survival and formation of connections in mouse ear and brain. Cell Tissue Res 341, 95-110 (2010).

14. Filova I, et al. Combined Atoh1 and Neurod1 Deletion Reveals Autonomous Growth of Auditory Nerve Fibers. Mol Neurobiol 57, 5307-5323 (2020).

15. Macova I, et al. Neurod1 Is Essential for the Primary Tonotopic Organization and Related Auditory Information Processing in the Midbrain. J Neurosci 39, 984-1004 (2019).

16. Dvorakova $M$, et al. Incomplete and delayed Sox2 deletion defines residual ear neurosensory development and maintenance. Sci Rep 6, 38253 (2016). 
17. Radde-Gallwitz K, Pan L, Gan L, Lin X, Segil N, Chen P. Expression of Islet1 marks the sensory and neuronal lineages in the mammalian inner ear. J Comp Neurol 477, 412-421 (2004).

865

866

867

868

869

870

871

872

873

874

875

876

877

878

879

880

881

882

883

884

885

886

887

888

889

890

891

892

893

894

895

896

897

898

899

900

901

902

903

904

905

18. Deng M, Yang H, Xie X, Liang G, Gan L. Comparative expression analysis of POU4F1, POU4F2 and ISL1 in developing mouse cochleovestibular ganglion neurons. Gene Expr Patterns 15, 31-37 (2014).

19. Chumak T, et al. Overexpression of IsI1 under the Pax2 Promoter, Leads to Impaired Sound Processing and Increased Inhibition in the Inferior Colliculus. Int J Mol Sci 22, (2021).

20. Petitpre $\mathrm{C}$, et al. Neuronal heterogeneity and stereotyped connectivity in the auditory afferent system. Nature communications 9,3691 (2018).

21. Li HJ, Kapoor A, Giel-Moloney M, Rindi G, Leiter AB. Notch signaling differentially regulates the cell fate of early endocrine precursor cells and their maturing descendants in the mouse pancreas and intestine. Dev Biol 371, 156-169 (2012).

22. Sun Y, Dykes IM, Liang X, Eng SR, Evans SM, Turner EE. A central role for Islet1 in sensory neuron development linking sensory and spinal gene regulatory programs. Nat Neurosci 11, 1283-1293 (2008).

23. Jahan I, Pan N, Kersigo J, Fritzsch B. Neurod1 suppresses hair cell differentiation in ear ganglia and regulates hair cell subtype development in the cochlea. PLoS One 5, e11661 (2010).

24. Li C, et al. Comprehensive transcriptome analysis of cochlear spiral ganglion neurons at multiple ages. Elife 9, (2020).

25. Stoeckli ET. Understanding axon guidance: are we nearly there yet? Development 145, (2018).

26. Fritzsch B, Kersigo J, Yang T, Jahan I, Pan N. Neurotrophic Factor Function During Ear Development: Expression Changes Define Critical Phases for Neuronal Viability. In: The Primary Auditory Neurons of the Mammalian Cochlea (eds Dabdoub A, Fritzsch B, Popper AN, Fay RR). Springer New York (2016).

27. Dennis DJ, Han S, Schuurmans C. bHLH transcription factors in neural development, disease, and reprogramming. Brain Res 1705, 48-65 (2019).

28. Yang T, Kersigo J, Wu S, Fritzsch B, Bassuk AG. Prickle1 regulates neurite outgrowth of apical spiral ganglion neurons but not hair cell polarity in the murine cochlea. PLoS One 12, e0183773 (2017). 
912

913

914

915

916

917

918

919

920

921

922

923

924

925

926

927

928

929

930

931

932

933

934

935

936

937

938

939

940

941

942

943

944

945

946

947

948

949

29. Bok J, Zenczak C, Hwang CH, Wu DK. Auditory ganglion source of Sonic hedgehog regulates timing of cell cycle exit and differentiation of mammalian cochlear hair cells. Proc Natl Acad Sci U S A 110, 13869-13874 (2013).

30. Shrestha BR, Chia C, Wu L, Kujawa SG, Liberman MC, Goodrich LV. Sensory Neuron Diversity in the Inner Ear Is Shaped by Activity. Cell 174, 1229-1246 e1217 (2018).

31. Pauley S, Wright TJ, Pirvola U, Ornitz D, Beisel K, Fritzsch B. Expression and function of FGF10 in mammalian inner ear development. Dev Dyn 227, 203-215 (2003).

32. Yu Y, et al. Sensorineural Hearing Loss and Mitochondrial Apoptosis of Cochlear Spiral Ganglion Neurons in Fibroblast Growth Factor 13 Knockout Mice. Frontiers in Cellular Neuroscience 15, (2021).

33. Fettiplace R, Hackney CM. The sensory and motor roles of auditory hair cells. Nature reviews Neuroscience 7, 19-29 (2006).

34. Neely S, Kim DO. Cochlear models incorporating active processes. Springer New York (2007).

35. Muller M, von Hunerbein K, Hoidis S, Smolders JW. A physiological place-frequency map of the cochlea in the CBA/J mouse. Hear Res 202, 63-73 (2005).

36. Zhou X, Jen PH, Seburn KL, Frankel WN, Zheng QY. Auditory brainstem responses in 10 inbred strains of mice. Brain Res 1091, 16-26 (2006).

37. Chambers AR, et al. Central Gain Restores Auditory Processing following Near-Complete Cochlear Denervation. Neuron 89, 867-879 (2016).

38. Harris JA, Rubel EW. Afferent regulation of neuron number in the cochlear nucleus: cellular and molecular analyses of a critical period. Hear Res 216-217, 127-137 (2006).

39. Lauer AM, Connelly CJ, Graham H, Ryugo DK. Morphological characterization of bushy cells and their inputs in the laboratory mouse (Mus musculus) anteroventral cochlear nucleus. PLoS One 8, e73308 (2013).

40. Karmakar K, et al. Hox2 Genes Are Required for Tonotopic Map Precision and Sound Discrimination in the Mouse Auditory Brainstem. Cell Rep 18, 185-197 (2017).

41. Fujiyama T, et al. Inhibitory and excitatory subtypes of cochlear nucleus neurons are defined by distinct bHLH transcription factors, Ptf1a and Atoh1. Development 136, 2049-2058 (2009).

42. Gruters KG, Groh JM. Sounds and beyond: multisensory and other non-auditory signals in the inferior colliculus. Front Neural Circuits 6, 96 (2012). 
950

951

952

953

954

955

956

957

958

959

960

961

962

963

964

965

966

967

968

969

970

971

972

973

974

975

976

977

978

979

980

981

982

983

984

985

986

987

988

989

990

991

992

993
43. Yeomans JS, Frankland PW. The acoustic startle reflex: neurons and connections. Brain Research Reviews 21, 301-314 (1995).

44. Koch M. The neurobiology of startle. Progress in neurobiology 59, 107-128 (1999).

45. Lee Y, Lopez DE, Meloni EG, Davis M. A primary acoustic startle pathway: obligatory role of cochlear root neurons and the nucleus reticularis pontis caudalis. J Neurosci 16, 3775-3789 (1996).

46. Swerdlow NR, Geyer MA, Braff DL. Neural circuit regulation of prepulse inhibition of startle in the rat: current knowledge and future challenges. Psychopharmacology 156, 194-215 (2001).

47. Fitch RH, Threlkeld SW, McClure MM, Peiffer AM. Use of a modified prepulse inhibition paradigm to assess complex auditory discrimination in rodents. Brain research bulletin $\mathbf{7 6 , 1 -}$ 7 (2008).

48. Hickox AE, Liberman MC. Is noise-induced cochlear neuropathy key to the generation of hyperacusis or tinnitus? J Neurophysiol 111, 552-564 (2014).

49. Pfaff SL, Mendelsohn M, Stewart CL, Edlund T, Jessell TM. Requirement for LIM homeobox gene IsI 1 in motor neuron generation reveals a motor neuron-dependent step in interneuron differentiation. Cell 84, 309-320 (1996).

50. Yang L, et al. IsI1Cre reveals a common Bmp pathway in heart and limb development. Development 133, 1575-1585 (2006).

51. Huber K, Narasimhan P, Shtukmaster S, Pfeifer D, Evans SM, Sun Y. The LIM-Homeodomain transcription factor Islet-1 is required for the development of sympathetic neurons and adrenal chromaffin cells. Dev Biol 380, 286-298 (2013).

52. Ahlgren U, Pfaff SL, Jessell TM, Edlund T, Edlund H. Independent requirement for ISL1 in formation of pancreatic mesenchyme and islet cells. Nature 385, 257-260 (1997).

53. Cai CL, et al. Isl1 identifies a cardiac progenitor population that proliferates prior to differentiation and contributes a majority of cells to the heart. Dev Cell 5, 877-889 (2003).

54. Liang $\mathrm{X}$, et al. IsI1 is required for multiple aspects of motor neuron development. Molecular and cellular neurosciences 47, 215-222 (2011).

55. Kania A, Jessell TM. Topographic motor projections in the limb imposed by LIM homeodomain protein regulation of ephrin-A:EphA interactions. Neuron 38, 581-596 (2003). 
994

995

996

997

998

999

1000

1001

1002

1003

1004

1005

1006

1007

1008

1009

1010

1011

1012

1013

1014

1015

1016

1017

1018

1019

1020

1021

1022

1023

1024

1025

1026

1027

1028

1029

1030

1031

1032

1033

1034

1035

1036

1037

56. Yamashita T, et al. High-resolution transcriptional dissection of in vivo Atoh1-mediated hair cell conversion in mature cochleae identifies Isl1 as a co-reprogramming factor. PLoS Genet 14, e1007552 (2018).

57. Chumak T, et al. Deterioration of the Medial Olivocochlear Efferent System Accelerates AgeRelated Hearing Loss in Pax2-IsI1 Transgenic Mice. Mol Neurobiol 53, 2368-2383 (2016).

58. Bohuslavova R, et al. Pax2-Islet1 Transgenic Mice Are Hyperactive and Have Altered Cerebellar Foliation. Mol Neurobiol 54, 1352-1368 (2017).

59. Huang M, Kantardzhieva A, Scheffer D, Liberman MC, Chen ZY. Hair cell overexpression of Islet1 reduces age-related and noise-induced hearing loss. J Neurosci 33, 15086-15094 (2013).

60. Morris JK, et al. A disorganized innervation of the inner ear persists in the absence of ErbB2. Brain Res 1091, 186-199 (2006).

61. Mao Y, Reiprich S, Wegner M, Fritzsch B. Targeted deletion of Sox10 by Wnt1-cre defects neuronal migration and projection in the mouse inner ear. PLoS One 9, e94580 (2014).

62. Geyer MA, Mcllwain KL, Paylor R. Mouse genetic models for prepulse inhibition: an early review. Mol Psychiatry 7, 1039-1053 (2002).

63. LeMasurier M, Gillespie PG. Hair-cell mechanotransduction and cochlear amplification. Neuron 48, 403-415 (2005).

64. Dallos $\mathrm{P}$, et al. Prestin-based outer hair cell motility is necessary for mammalian cochlear amplification. Neuron 58, 333-339 (2008).

65. Herranen A, et al. Deficiency of the ER-stress-regulator MANF triggers progressive outer hair cell death and hearing loss. Cell death \& disease 11, 100 (2020).

66. Liberman LD, Liberman MC. Cochlear Efferent Innervation Is Sparse in Humans and Decreases with Age. J Neurosci 39, 9560-9569 (2019).

67. Hafidi A. Peripherin-like immunoreactivity in type II spiral ganglion cell body and projections. Brain Res 805, 181-190 (1998).

68. Elliott KL, et al. Developmental Changes in Peripherin-eGFP Expression in Spiral Ganglion Neurons. Front Cell Neurosci 15, 678113 (2021).

69. Zhang KD, Coate TM. Recent advances in the development and function of type II spiral ganglion neurons in the mammalian inner ear. Seminars in cell \& developmental biology 65 , 80-87 (2017). 
70. Froud KE, et al. Type II spiral ganglion afferent neurons drive medial olivocochlear reflex suppression of the cochlear amplifier. Nature communications 6, 7115 (2015).

71. Maison S, Liberman LD, Liberman MC. Type II Cochlear Ganglion Neurons Do Not Drive the Olivocochlear Reflex: Re-Examination of the Cochlear Phenotype in Peripherin Knock-Out Mice. eNeuro 3, (2016).

72. Matei $\mathrm{V}$, et al. Smaller inner ear sensory epithelia in Neurog 1 null mice are related to earlier hair cell cycle exit. Dev Dyn 234, 633-650 (2005).

73. Pauley S, Lai E, Fritzsch B. Foxg1 is required for morphogenesis and histogenesis of the mammalian inner ear. Dev Dyn 235, 2470-2482 (2006).

74. Nichols DH, Pauley S, Jahan I, Beisel KW, Millen KJ, Fritzsch B. Lmx1a is required for segregation of sensory epithelia and normal ear histogenesis and morphogenesis. Cell Tissue Res 334, 339-358 (2008).

75. Nichols $\mathrm{DH}$, et al. Interaction with ectopic cochlear crista sensory epithelium disrupts basal cochlear sensory epithelium development in Lmx1a mutant mice. Cell Tissue Res 380, 435448 (2020).

76. Pavlinkova G. Molecular Aspects of the Development and Function of Auditory Neurons. Int J Mol Sci 22, (2020).

77. Zine A, Messat $Y$, Fritzsch B. A human induced pluripotent stem cell-based modular platform to challenge sensorineural hearing loss. Stem Cells, (2021).

78. Hobert O, Westphal H. Functions of LIM-homeobox genes. Trends Genet 16, 75-83 (2000).

79. Mamanova L, Miao Z, Jinat A, Ellis P, Shirley L, Teichmann SA. High-throughput full-length single-cell RNA-seq automation. Nat Protoc 16, 2886-2915 (2021).

80. Dobin A, et al. STAR: ultrafast universal RNA-seq aligner. Bioinformatics 29, 15-21 (2013).

81. Martin M. Cutadapt removes adapter sequences from high-throughput sequencing reads. 2011 17, 3 (2011).

82. Bolger AM, Lohse M, Usadel B. Trimmomatic: a flexible trimmer for Illumina sequence data. Bioinformatics 30, 2114-2120 (2014).

83. Kopylova E, Noe L, Touzet H. SortMeRNA: fast and accurate filtering of ribosomal RNAs in metatranscriptomic data. Bioinformatics 28, 3211-3217 (2012). 
1081

1082

1083

1084

1085

1086

1087

1088

1089

1090

1091

1092

1093

1094

1095

1096

1097

1098

1099

1100

1101

1102

1103

1104

1105

1106

1107

1108

1109

1110

1111

84. Love MI, Huber W, Anders S. Moderated estimation of fold change and dispersion for RNAseq data with DESeq2. Genome Biol 15, 550 (2014).

85. Boyle El, et al. GO::TermFinder--open source software for accessing Gene Ontology information and finding significantly enriched Gene Ontology terms associated with a list of genes. Bioinformatics 20, 3710-3715 (2004).

86. Raudvere $\mathrm{U}$, et al. g:Profiler: a web server for functional enrichment analysis and conversions of gene lists (2019 update). Nucleic acids research 47, W191-W198 (2019).

87. Shannon $\mathrm{P}$, et al. Cytoscape: a software environment for integrated models of biomolecular interaction networks. Genome Res 13, 2498-2504 (2003).

88. Merico D, Isserlin R, Stueker O, Emili A, Bader GD. Enrichment map: a network-based method for gene-set enrichment visualization and interpretation. PLoS One 5, e13984 (2010).

89. Fritzsch B, Duncan JS, Kersigo J, Gray B, Elliott KL. Neuroanatomical Tracing Techniques in the Ear: History, State of the Art, and Future Developments. In: B. Sokolowski, Ed: Auditory and Vestibular Research: Methods and Protocols). Springer Science+Business Media New York (2016).

90. Simmons D, Duncan J, de Caprona DC, Fritzsch B. Development of the inner ear efferent system. In: Auditory and vestibular efferents). Springer (2011).

91. Bures Z, Grécová J, Popelár J, Syka J. Noise exposure during early development impairs the processing of sound intensity in adult rats. The European journal of neuroscience 32, 155-164 (2010). 